\title{
PAIRS OF SELFADJOINT OPERATORS AND THEIR INVARIANTS
}

\author{
D. ALPAY AND I. GOHBERG
}

Dedicated to Mikhail Birman on the occasion of his 75th birthday, with admiration

\begin{abstract}
A trace formula is proved for pairs of selfadjoint operators that are close to each other in a certain sense. An important role is played by a function analytic in the open upper half-plane and with positive imaginary part there. This function, called the characteristic function of the pair, coincides with Krein's $Q$-function in the case where the selfadjoint operators are canonical extensions of a common simple and closed Hermitian operator. Special emphasis is given to the finite-dimensional case. Relationships with Krein's spectral shift function are also considered. Finally, the case of canonical differential expressions is discussed briefly. In this case, the function $N$ may be chosen to be the Weyl function of the canonical differential expression.
\end{abstract}

\section{§1. INTRODUCTION}

This paper is the second in a series where we study models and trace formulas for pairs of selfadjoint operators. In 8 we considered the case of the so-called canonical differential expressions (see $\$ 6$ and equation (6.1) for the definitions). Here our starting point is a pair $\left(H_{+}, H_{-}\right)$of selfadjoint operators defined on a separable Hilbert space $\mathcal{H}$ and possessing the following properties:

1) for all $\omega \in \mathbb{C} \backslash \mathbb{R}$,

$$
\operatorname{rank}\left(\left(H_{+}-\omega I\right)^{-1}-\left(H_{-}-\omega I\right)^{-1}\right)=n<\infty ;
$$

2) we have

$$
\bigcap_{\omega \in \mathbb{C} \backslash \mathbb{R}} \operatorname{ker}\left(\left(H_{+}-\omega I\right)^{-1}-\left(H_{-}-\omega I\right)^{-1}\right)=\{0\} .
$$

The first condition expresses the fact that the operators $H_{+}$and $H_{-}$are close to each other. The second condition means that $\{0\}$ is the only closed subspace of $\mathcal{H}$ invariant under $H_{+}$and $H_{-}$on which $H_{+}$and $H_{-}$coincide.

A model for such a pair was given by L. de Branges and J. Rovnyak in 21. In fact, these authors considered the more general case where the operators

$$
\left(H_{+}-\omega I\right)^{-1}-\left(H_{-}-\omega I\right)^{-1}
$$

are of trace class for $\omega \in \mathbb{C} \backslash \mathbb{R}$. If these operators are of finite rank (say $n$ ), the result of de Branges and Rovnyak can be formulated in terms of a $\mathbb{C}^{n \times n}$-valued function analytic in the open upper half-plane $\mathbb{C}_{+}$and with a positive real part there, and we rephrase it in terms of a function with a positive imaginary part in the open upper half-plane $\mathbb{C}_{+}$ (i.e., a Herglotz-Nevanlinna function). Before stating the result, we need a definition.

2000 Mathematics Subject Classification. Primary 34L25, 81U40, 47A56.

Key words and phrases. Krel̆n's spectral shift function, the $Q$-function associated with a symmetric operator, the Weyl function.

The research of the second author was supported by the Israel Science Foundation (grant no. 322/00). 
If a Herglotz-Nevanlinna function $N$ is extended to the lower half-plane by the formula $N(z)=N\left(z^{*}\right)^{*}$, then the functions

$$
K_{N}(z, \omega)=\frac{N(z)-N(\omega)^{*}}{4 \pi\left(z-\omega^{*}\right)} \quad \text { and } \quad K_{-N^{-1}}(z, \omega)=\frac{-N(z)^{-1}+N(\omega)^{-*}}{4 \pi\left(z-\omega^{*}\right)}
$$

are positive in the sense of reproducing kernels in $\mathbb{C} \backslash \mathbb{R}$; see [43, 11] for the definition of positivity. Thus, we can consider the associated reproducing-kernel Hilbert spaces of functions analytic in $\mathbb{C} \backslash \mathbb{R}$ with reproducing kernels $K_{N}(z, \omega)$ and $K_{-N^{-1}}(z, \omega)$, respectively. We shall denote these spaces by $\mathcal{L}(N)$ and $\mathcal{L}\left(-N^{-1}\right)$. For $N$ arising from such a pair $\left(H_{+}, H_{-}\right)$, the spaces $\mathcal{L}(N)$ and $\mathcal{L}\left(-N^{-1}\right)$ provide a model for the pair $\left(H_{+}, H_{-}\right)$, as is explicitly seen in the following result due to de Branges and Rovnyak (see [21]). Two proofs of this result will be given in the paper; see Subsection 2.4 for the case of matrices and Subsection 5.1 for the case of unbounded operators.

Theorem 1.1. Let $\left(H_{+}, H_{-}\right)$be a pair of selfadjoint operators defined on a separable Hilbert space and with the following two properties:

1) there is $n \in \mathbb{N}$ such that

$$
\operatorname{dim} \operatorname{ran}\left(\left(H_{+}-\omega I\right)^{-1}-\left(H_{-}-\omega I\right)^{-1}\right)=n
$$

for all $\omega \in \mathbb{C} \backslash \mathbb{R}$

2) we have

$$
\bigcap_{\omega \in \mathbb{C} \backslash \mathbb{R}} \operatorname{ker}\left(\left(H_{+}-\omega I\right)^{-1}-\left(H_{-}-\omega I\right)^{-1}\right)=\{0\} .
$$

Then there exists a $\mathbb{C}^{n \times n}$-valued Herglotz-Nevanlinna function with $\operatorname{det} N \neq 0$ and with the following properties:

1) there are unitary transformations

$$
U_{+}: \mathcal{H} \rightarrow \mathcal{L}(N) \quad \text { and } \quad U_{-}: \mathcal{H} \rightarrow \mathcal{L}\left(-N^{-1}\right)
$$

such that for $\omega \in \mathbb{C} \backslash \mathbb{R}$ we have

$$
\begin{aligned}
& U_{+}\left(H_{+}-\omega I\right)^{-1} f=R_{\omega} U_{+} f, \\
& U_{-}\left(H_{-}-\omega I\right)^{-1} f=R_{\omega} U_{-} f,
\end{aligned}
$$

where $R_{\omega}$ is the resolvent-like operator defined by $R_{\omega} f(z)=\frac{f(z)-f(\omega)}{z-\omega}$;

2) for every $f \in \mathcal{H}$ we have

$$
\mathcal{M}_{-N^{-1}} U_{+} f=U_{-} f
$$

where $\mathcal{M}_{-N^{-1}}$ denotes the operator of left multiplication by $-N^{-1}$.

As a consequence of the construction of de Branges and Rovnyak, we obtain the following statement.

Theorem 1.2. Let $\left(H_{+}, H_{-}\right)$be a pair of selfadjoint operators defined on a separable Hilbert space and satisfying the hypothesis mentioned above. Let $N$ be the associated Herglotz-Nevanlinna function. Then for all $\omega$ in the open upper half-plane we have

$$
\operatorname{Tr}\left\{\left(H_{+}-\omega I\right)^{-1}-\left(H_{-}-\omega I\right)^{-1}\right\}=\operatorname{Tr} N(\omega)^{-1} N^{\prime}(\omega)
$$

and

$$
\widetilde{\operatorname{det}}_{z_{0}}\left(H_{-}-z I\right)\left(H_{+}-z I\right)^{-1}=\operatorname{det} N(z) N\left(z_{0}\right)^{-1}, \quad z \in \mathbb{C}_{+},
$$

where $z_{0} \in \mathbb{C} \backslash \mathbb{R}$ and $\widetilde{\operatorname{det}_{z_{0}}}$ is the generalized perturbation determinant associated with the pair $\left(H_{+}, H_{-}\right)$. 
We also study relationships between the trace formulas and the spectral shift function introduced by I. M. Lifshifts in [40, 41] and by M. G. Krĕn (see [33, 17]). This function is such that

$$
\operatorname{Tr}\left(r\left(H_{+}\right)-r\left(H_{-}\right)\right)=\int_{\mathbb{R}} r^{\prime}(t) \xi(t) d t
$$

for all rational functions analytic at infinity and with poles in $\sigma\left(H_{+}\right) \cap \sigma\left(H_{-}\right)$. See [32] (3), p. 138]. The choice $r(t)=\frac{1}{t-\omega}$ with $\omega$ off the real line leads to the formula

$$
\operatorname{Tr}\left(\left(H_{+}-\omega I\right)^{-1}-\left(H_{-}-\omega I\right)^{-1}\right)=-\int_{\mathbb{R}} \frac{\xi(t) d t}{(t-\omega)^{2}} .
$$

It is of interest to find explicit examples where the hypotheses of Theorem 1.1 are fulfilled and, consequently, the above trace formula is true. As has already been mentioned, one such example is given by the case of canonical differential expressions; see $\sqrt{6}$ and 8]. Another example is when $H_{+}$and $H_{-}$are extensions of a common Hermitian operator with deficiency indices $(n, n)$ (see [23, p. 420]). In this case we use the notion of the $Q$-function of a closed Hermitian operator (due to Kreı̆n; see [30, 31, 36, 42]).

The paper consists of six sections besides the Introduction. In $\S 2$ we focus on the finitedimensional case, and give a self-contained exposition based on the realization theory of matrix-valued rational functions and on the theory of finite-dimensional reproducingkernel Hilbert spaces. In $\S 3$ we study infinite-dimensional $\mathcal{L}(N)$-spaces. $\S 4$ is devoted to the case of pairs of bounded selfadjoint operators. In $\S 5$ we consider the case of possibly unbounded operators. In the last two sections we consider two special cases: canonical differential expressions $(\S 6)$ and selfadjoint extensions of a Hermitian operator ( $(7)$.

In conclusion, we note that this paper has a number of links with the work of de Branges and Rovnyak and with the work of Kreı̆ and Langer. These relationships are demonstrated in the text.

\section{§2. The Finite-Dimensional CASE}

In this section we focus on the finite-dimensional case, that is, we consider the case of pairs of selfadjoint matrices. We define an invariant for such a pair, which we call the characteristic function of the pair. Because of the finite-dimensionality hypothesis, the arguments do not involve difficult analytic tools. Realization of rational matrix-valued functions plays an important role in the theory; see [14]. We also use the canonical form of a matrix that is Hermitian with respect to an indefinite inner product; see [28, 29, 44].

2.1. Finite-dimensional reproducing-kernel Hilbert spaces. The theory of reproducing-kernel Hilbert spaces is used extensively in this paper; here we review the main features of the finite-dimensional case.

A Hilbert space $\mathcal{H}$ of $\mathbb{C}^{n}$-valued functions defined on a set $\Omega$ is called a reproducingkernel Hilbert space if there exists a $\mathbb{C}^{n \times n}$-valued function $K(z, w)$ of two variables with the following two properties: first, the function $z \mapsto K(z, w) e$ belongs to $\mathcal{H}$ for every $w \in \Omega$ and every $e \in \mathbb{C}^{n}$, and next, we have

$$
\langle f(\cdot), K(\cdot, w) e\rangle_{\mathcal{H}}=e^{*} f(w)
$$

for every $f \in \mathcal{H}$.

The function $K(z, w)$ is called the reproducing kernel of the space. It is unique. By Riesz's representation theorem for bounded functionals in a Hilbert space, a Hilbert space of functions has a reproducing kernel if and only if the point evaluation functionals $f \mapsto e^{*} f(w)$ are bounded (here $e \in \mathbb{C}^{n}$ and $w \in \Omega$ ).

Property (2.1) implies the following statement. 
D. ALPAY AND I. GOHBERG

Lemma 2.1. Let $\mathcal{H}$ be a reproducing-kernel Hilbert space of $\mathbb{C}^{n}$-valued functions defined on a set $\Omega$, and let $K(z, w)$ be the reproducing kernel of $\mathcal{H}$. Then the linear span of the functions $z \mapsto K(z, w)$ e as $w$ varies in $\Omega$ and e varies in $\mathbb{C}^{n}$ is dense in $\mathcal{H}$. Furthermore,

$$
\langle K(\cdot, v) d, K(\cdot, w) e\rangle_{\mathcal{H}}=e^{*} K(w, v) d \quad \text { for all } v, w \in \Omega \text { and all } d, e \in \mathbb{C}^{n} .
$$

Proof. By (2.1), a function $f \in \mathcal{H}$ orthogonal to all the functions $z \mapsto K(z, w) e$ satisfies $e^{*} f(w)=0$ for all $w \in \Omega$ and all $e \in \mathbb{C}^{n}$, whence $f \equiv 0$. This proves the first claim. To prove the second, we apply (2.1) to $f(z)=K(z, v) d$ and obtain (2.2).

If $\mathcal{H}$ is finite-dimensional, then the point evaluation functionals $f \mapsto e^{*} f(w)$ (with $e \in \mathbb{C}^{n}$ and $w \in \Omega$ ) are bounded automatically, and $\mathcal{H}$ is a reproducing-kernel Hilbert space. The linear span of the functions $z \mapsto K(z, w) e$ for $w \in \Omega$ and $e \in \mathbb{C}^{n}$ is not merely dense in $\mathcal{H}$ but is equal to $\mathcal{H}$. Now we recall a formula for the reproducing kernel in the finite-dimensional case. Let $m=\operatorname{dim} \mathcal{H}$, and let $F$ be a $\mathbb{C}^{n \times m}$-valued function whose columns form a basis of $\mathcal{H}$. The inner product $\langle\cdot, \cdot\rangle_{\mathcal{H}}$ determines a strictly positive matrix $P \in \mathbb{C}^{m \times m}$ (called the Gram matrix) via the relation

$$
\langle F c, F d\rangle_{\mathcal{H}}=d^{*} P c, \quad c, d \in \mathbb{C}^{m} .
$$

Lemma 2.2. The reproducing kernel of $\mathcal{H}$ is given by the formula

$$
K(z, w)=F(z) P^{-1} F(w)^{*} .
$$

Proof. We must check the two conditions mentioned at the beginning of the section. The first condition is clear because $K(z, w) e=F(z)\left(P^{-1} F(w)^{*} e\right)$. To prove the second, write $f(z)=F(z) d$, where $d \in \mathbb{C}^{m}$. Then

$$
\begin{aligned}
\langle f(\cdot), K(\cdot, w) e\rangle_{\mathcal{H}} & =\left\langle F(\cdot) d, F(\cdot) P^{-1} F(w)^{*} e\right\rangle_{\mathcal{H}} \\
& =\left(P^{-1} F(w)^{*} e\right)^{*} P d=e^{*} F(w) d=e^{*} f(w) .
\end{aligned}
$$

Formula (2.3) has the following consequence.

Corollary 2.3. Assume that $\Omega \subset \mathbb{C}$ and that the elements of $\mathcal{H}$ are rational functions. Then for every $w \in \Omega$ the reproducing kernel $K(z, w)$ is a rational function in $z$.

Now we discuss an important case where the elements of $\mathcal{H}$ are rational. For $\alpha \in \mathbb{C}$ and $f$ a matrix-valued function analytic in a neighborhood of $\alpha$, we define

$$
R_{\alpha} f(z)= \begin{cases}\frac{f(z)-f(\alpha)}{z-\alpha} & \text { if } z \neq \alpha, \\ f^{\prime}(\alpha) & \text { if } z=\alpha .\end{cases}
$$

The operators $R_{\alpha}$ satisfy the resolvent equation

$$
R_{\alpha}-R_{\beta}=(\alpha-\beta) R_{\alpha} R_{\beta} .
$$

Let $\alpha \in \mathbb{C}$, and let $\mathcal{H}$ be an $R_{\alpha}$-invariant finite-dimensional space of $\mathbb{C}^{n}$-valued functions analytic in a neighborhood of $\alpha$. Then the elements of $\mathcal{H}$ are rational functions without a pole at the point $\alpha$. Indeed, suppose $F(z)$ is as in Lemma 2.2 that is, a $\mathbb{C}^{n \times m}$-valued function whose columns form a basis of $\mathcal{H}$. Let $f_{j}$ be the $j$ th column of $F$. By $R_{\alpha}$-invariance, there exists a vector $a_{j} \in \mathbb{C}^{m}$ such that $R_{\alpha} f_{j}=F a_{j}$. Let $A=\left(a_{1} a_{2} \cdots a_{m}\right) \in \mathbb{C}^{m \times m}$. We have $R_{\alpha} F=F A$. Thus,

$$
\frac{F(z)-F(\alpha)}{z-\alpha}=F(z) A \text {, }
$$

whence

$$
F(z)=F(\alpha)\left(I_{m}-(z-\alpha) A\right)^{-1},
$$

which is a rational matrix-valued function analytic at the point $\alpha$. 
We note that the material presented in this section is well known and the proofs have been provided for completeness. For instance, Lemma 2.2] can be found in [25] and [43]. We also refer to [25, 43] for more information on reproducing-kernel Hilbert spaces.

2.2. Finite-dimensional $\mathcal{L}(N)$-spaces. In this paper we shall be particularly interested in reproducing-kernel Hilbert spaces whose reproducing kernel is of the form

$$
K_{N}(z, w)=\frac{N(z)-N(w)^{*}}{z-\bar{w}}
$$

where $N$ is a $\mathbb{C}^{n \times n}$-valued function analytic in $\mathbb{C} \backslash \mathbb{R}$. These spaces were introduced by de Branges; see [18, 19. We shall denote by $\mathcal{L}(N)$ the associated reproducing-kernel Hilbert space. Now we review the main features of these spaces in the finite-dimensional case. We make use of the realization theory of matrix-valued functions. The infinite-dimensional case will be considered in $\$ 3$

Lemma 2.1 and the finite-dimensionality hypothesis imply that the functions of the form $z \mapsto \frac{N(z)-N(w)^{*}}{z-\bar{w}} e$ span $\mathcal{L}(N)$ when $w$ varies in $\mathbb{C} \backslash \mathbb{R}$ and $e$ varies in $\mathbb{C}^{n}$. Furthermore,

$$
\left\langle\frac{N(z)-N(v)^{*}}{z-\bar{v}} d, \frac{N(z)-N(w)^{*}}{z-\bar{w}} e\right\rangle_{\mathcal{L}(N)}=e^{*} \frac{N(w)-N(v)^{*}}{w-\bar{v}} d
$$

for all $v, w \in \mathbb{C} \backslash \mathbb{R}$ and all $d, e \in \mathbb{C}^{n}$.

Equation (2.6) does not take the special form of the kernel into account. In the sequel we give more precise descriptions of the inner product in $\mathcal{L}(N)$; see Proposition 2.6 for the finite-dimensional case and Theorem 3.2 for the general case.

Lemma 2.4. Let $N$ be a $\mathbb{C}^{n \times n}$-valued function analytic in $\mathbb{C}_{+}$. Suppose that the kernel $K_{N}(z, w)$ is positive in $\mathbb{C}_{+}$and that the corresponding space $\mathcal{L}(N)$ is finite-dimensional. Then $N$ is rational, $N(z)=N(\bar{z})^{*}$ for $z \in \mathbb{C} \backslash \mathbb{R}$, and $\operatorname{Im} N(z) \geq 0$ for $z \in \mathbb{C}_{+}$.

Proof. Since $N(w)^{*}=N(\bar{w})$, we have

$$
K_{N}(z, w)=\frac{N(z)-N(\bar{w})}{z-\bar{w}}=\left(R_{\bar{w}} N\right)(z) .
$$

Therefore, using the resolvent identity (2.4), we obtain

$$
R_{\alpha} K_{N}(z, w)=\frac{1}{\alpha-\bar{w}}\left\{K_{N}(z, \bar{\alpha})-K_{N}(z, w)\right\} .
$$

Since $\mathcal{L}(N)$ is finite-dimensional, the functions $z \mapsto K(z, w)$ e span the entire space $\mathcal{L}(N)$ when $w$ runs through $\mathbb{C} \backslash \mathbb{R}$ and $e$ runs through $\mathbb{C}^{n}$, so that $\mathcal{L}(N)$ is $R_{\alpha}$-invariant. Therefore, this space consists of rational functions. Next, Lemma 2.2 shows that $z \mapsto$ $K_{N}(z, w)$ is rational. In particular, $\bar{w}$ is a removable singularity of $z \mapsto K_{N}(z, w)$ and, thus, $N(\bar{w})=N(w)^{*}$. To see that $\operatorname{Im} N(z) \geq 0$ for $z \in \mathbb{C}_{+}$, it suffices to observe that $K_{N}(z, z)$ is a positive matrix for $z \in \mathbb{C} \backslash \mathbb{R}$. Finally, $N$ is rational because of the formula

$$
N(z)=N\left(w_{0}\right)^{*}+\left(z-\overline{w_{0}}\right) K_{N}\left(z, w_{0}\right),
$$

where $w_{0}$ is any preassigned point in $\mathbb{C}_{+}$.

Now we describe the minimal realizations of the matrix-valued functions analytic at infinity and such that $N(z)=N(\bar{z})^{*}$. We studied such realizations in [3]. We refer to [14] for the background on the various notions and results from the theory of realization of rational matrix-valued functions used in the sequel.

Proposition 2.5. Let $N$ be $a \mathbb{C}^{n \times n}$-valued rational function analytic at infinity, and let $N(z)=D+C\left(z I_{m}-A\right)^{-1} B$ be a minimal realization of $N$. Then $N$ satisfies the 
D. ALPAY AND I. GOHBERG

condition $N(z)=N(\bar{z})^{*}$ if and only if the following conditions are fulfilled: $D=D^{*}$ and there exists a Hermitian invertible matrix $H$ such that

$$
\begin{aligned}
A H & =H A^{*}, \\
-C H & =B^{*} .
\end{aligned}
$$

Furthermore, we have

$$
\frac{N(z)-N(w)^{*}}{z-\bar{w}}=C\left(z I_{m}-A\right)^{-1} H\left(w I_{m}-A\right)^{-*} C^{*},
$$

and the following statements are equivalent:

1) the imaginary part of $N$ is positive in the open upper half-plane;

2) the matrix $H$ is strictly positive;

3) the kernel $K_{N}(z, w)$ is positive in $\mathbb{C} \backslash \sigma(A)$.

Proof. A minimal realization of the rational function $N(\bar{z})^{*}$ is given by

$$
N(\bar{z})^{*}=D^{*}+B^{*}\left(z I_{m}-A^{*}\right)^{-1} C^{*} .
$$

Since $N(z)=N(\bar{z})^{*}$, we have

$$
D+C\left(z I_{m}-A\right)^{-1} B=D^{*}+B^{*}\left(z I_{m}-A^{*}\right)^{-1} C^{*} .
$$

Thus, there is a unique matrix $H$ such that

$$
\left(\begin{array}{ll}
A & B \\
C & D
\end{array}\right)\left(\begin{array}{cc}
-H & 0 \\
0 & I_{n}
\end{array}\right)=\left(\begin{array}{cc}
-H & 0 \\
0 & I_{n}
\end{array}\right)\left(\begin{array}{ll}
A^{*} & C^{*} \\
B^{*} & D^{*}
\end{array}\right) .
$$

Taking the adjoint of (2.11), we see that $H^{*}$ is also a solution of (2.11) and thus $H=H^{*}$. Furthermore, (2.11) is equivalent to $D=D^{*}$ and (2.8)-2.9).

We prove (2.10). Observe that

$$
N(z)=D-C\left(z I_{m}-A\right)^{-1} H C^{*} .
$$

Thus,

$$
\begin{aligned}
N(z) & -N(w)^{*}=N(z)-N(\bar{w}) \\
& =C\left\{-\left(z I_{m}-A\right)^{-1}+\left(\bar{w} I_{m}-A\right)^{-1}\right\} H C^{*} \\
& =C\left(z I_{m}-A\right)^{-1}\left(\bar{w} I_{m}-A\right)^{-1} H C^{*} \cdot(z-\bar{w}) .
\end{aligned}
$$

By (2.8), we have

$$
\left(\bar{w} I_{m}-A\right)^{-1} H=H\left(\bar{w} I_{m}-A^{*}\right)^{-1}
$$

and we arrive at (2.10).

Now we turn to the proof of the equivalence of claims 1)-3) in the theorem. Formula (2.10) shows that 2) implies 3). The fact that 3) implies 1) is trivial (simply take $z=w$ ). It remains to show that 1) implies 2). First, we note that the function $N$ is analytic in the open upper half-plane $\mathbb{C}_{+}$. Indeed, the function $S(z)=\left(I_{n}+i N(z)\right)\left(I_{n}-i N(z)\right)^{-1}$ is contractive at all points where it is defined in $\mathbb{C}_{+}$; consequently, it admits analytic and contractive extension to $\mathbb{C}_{+}$, so that $N$ is analytic in $\mathbb{C}_{+}$. Thus, this function is analytic in $\mathbb{C} \backslash \mathbb{R}$ because $N(z)=N(\bar{z})^{*}$. It follows that the matrix $A$ has spectrum only on the real line. Therefore, the pair $(A, H)$ has a canonical representation of the form $\bigoplus_{k=1}^{M}\left(J_{m_{k}}\left(\lambda_{k}\right), \epsilon_{k} E_{m_{k}}\right)$, where $\lambda_{k}$ is a real number for every $k \in\{1, \ldots, M\}, J_{m_{k}}\left(\lambda_{k}\right)$ is 
the $\left(m_{k} \times m_{k}\right)$-Jordan block with eigenvalue $\lambda_{k}, E_{n_{k}}$ is the matrix with the entries on the main antidiagonal equal to 1 and all other entries equal to 0 :

$$
J_{n_{k}}\left(\lambda_{k}\right)=\left(\begin{array}{cccccc}
\lambda_{k} & 1 & 0 & 0 & \cdots & 0 \\
0 & \lambda_{k} & 1 & 0 & \cdots & 0 \\
\cdot & \cdot & & & \cdots & \\
0 & \cdot & & & \cdots & 1 \\
0 & \cdot & & & & \lambda_{k}
\end{array}\right), \quad E_{n_{k}}=\left(\begin{array}{ccccc}
0 & \cdots & 0 & 0 & 1 \\
0 & \cdots & 0 & 1 & 0 \\
0 & \cdots & 1 & 0 & 0 \\
. & \cdot & & & 0 \\
1 & 0 & & &
\end{array}\right),
$$

and $\epsilon_{k} \in\{-1,1\}$.

The remaining part of the proof that 1) implies 2) is split in several steps.

Step 1. Let $\lambda \in \mathbb{R}$, and let $\ell \geq 2$. The function $\operatorname{Im} \frac{1}{(z-\lambda)^{\ell}}$ takes both positive and negative values in the open upper half-plane.

We set $z=\lambda+\rho e^{i \theta}$, where $\rho>0$ and $\theta \in(0, \pi)$. Then

$$
\operatorname{Im} \frac{1}{(z-\lambda)^{\ell}}=-\frac{2 \sin (\ell \theta)}{\rho^{\ell}} .
$$

For $\ell \geq 2$ the argument $\ell \theta$ belongs to $(0,2 \pi)$; thus, $\sin (\ell \theta)$ takes both positive and negative values.

Step 2. Assume that $(A, H)$ consists only of an elementary block, that is, $M=1$ in the above canonical form. Then $m_{1}=1$, and 1) implies 2).

We set $m_{1}=m$ and assume that $m \geq 2$ and $A=J(\lambda) \in \mathbb{C}^{m \times m}$. We write

$$
A=J(\lambda)=\lambda I_{m}+T,
$$

where $T^{m+1}=0$. Then

$$
\begin{aligned}
N(z) & =I_{n}-C\left(z I_{m}-A\right)^{-1} H C^{*} \\
& =I_{n}-C\left((z-\lambda) I_{m}-T\right)^{-1} H C^{*} \\
& =I_{n}-\sum_{k=0}^{m} \frac{C T^{k} H C^{*}}{(z-\lambda)^{k+1}} .
\end{aligned}
$$

Since $A H=H A^{*}$, we have $T H=H T^{*}$ and $T^{\ell} H=H T^{* \ell}$ for all $\ell \geq 1$. Therefore,

$\operatorname{Im} N(z)=-C H C^{*}\left\{\frac{1}{z-\lambda}-\frac{1}{\bar{z}-\lambda}\right\}-\cdots-C T^{m} H C^{*}\left\{\frac{1}{(z-\lambda)^{m+1}}-\frac{1}{(\bar{z}-\lambda)^{m+1}}\right\}$.

We set $z=\lambda+\rho e^{i \theta}$ as at the preceding step and multiply both sides of (2.12) by $\rho^{m+1}$. Letting $\rho \rightarrow 0$, we see that

$$
0 \leq \lim _{\rho \rightarrow 0} \rho^{m} \operatorname{Im} N\left(\lambda+\rho e^{i \theta}\right)=(2 \sin (m+1) \theta) C T^{m} H C^{*} .
$$

Consequently, $C T^{m} C^{*}=0$.

Now, multiplying (2.12) by $\rho^{m}$ and letting $\rho \rightarrow 0$, we show that $C T^{m-1} H C^{*}=0$. Reiterating, we obtain

$$
C T H C^{*}=C T^{2} H C^{*}=\cdots=C T^{m} H C^{*}=0 .
$$

Thus,

$$
\operatorname{Im} N(z)=-C H C^{*}\left\{\frac{1}{z-\lambda}-\frac{1}{\bar{z}-\lambda}\right\},
$$


and we have $C H C^{*} \geq 0$. This inequality and (2.13) imply the relation

$$
\left(\begin{array}{c}
C \\
C A \\
\vdots \\
C A^{n}
\end{array}\right) H\left(C^{*} A^{*} C^{*} \cdots A^{* n} C^{*}\right)=\left(\begin{array}{c}
I_{m} \\
\lambda I_{m} \\
\vdots \\
\lambda^{n} I_{m}
\end{array}\right) C H C^{*}\left(I_{m} \bar{\lambda} I_{m} \cdots \overline{\lambda^{n}} I_{m}\right) \geq 0
$$

because $T^{\ell}=0$ for $\ell \geq m+1$. Since the pair $(C, A)$ is observable, the pair $\left(A^{*}, C^{*}\right)$ is controllable:

$$
\operatorname{ran}\left(C^{*} A^{*} C^{*} \cdots A^{* n} C^{*}\right)=\mathbb{C}^{n}
$$

and (2.14) implies that $H \geq 0$.

Step 3. Now we turn to the case where in the canonical representation of the pair $(A, H)$ there is a unique eigenvalue $\lambda$ corresponding to possibly different blocks:

$$
(A, H)=\bigoplus\left(J_{i}(\lambda), H_{i}\right)
$$

where both $J_{i}(\lambda)$ and $H_{i}$ are in $\mathbb{C}^{m_{i} \times m_{i}}$. We write $J_{i}(\lambda)=\lambda I_{m_{i}}+T_{i}$, where $T_{i}^{m_{i}+1}=0$.

Let $C=\left(C_{1} C_{2} \ldots\right)$ be the corresponding matrix representation of $C$. Arguing as at Step 2, we obtain

$$
\sum_{i} C_{i} T_{i}^{n} H_{i} C_{i}^{*}=0, \quad m=1,2, \ldots, \sup m_{i}
$$

and

$$
\sum_{i} C_{i} H_{i} C_{i}^{*} \geq 0
$$

Set $M=\sup _{i} m_{i}$. The counterpart of (2.14) looks like this:

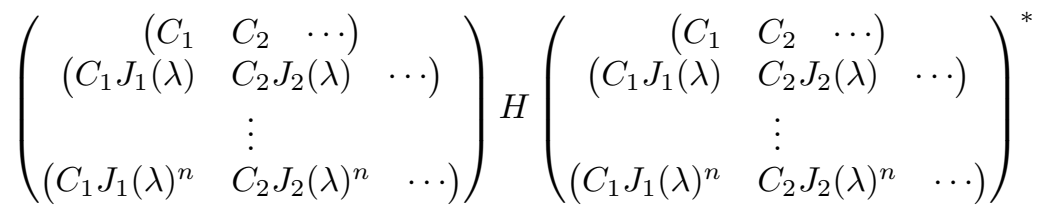

$$
\begin{aligned}
& =\left(\begin{array}{c}
I_{m} \\
\lambda I_{m} \\
\vdots \\
\lambda^{n} I_{m}
\end{array}\right) \sum_{i} C_{i} H_{i} C_{i}^{*}\left(I_{m} \bar{\lambda} I_{m} \cdots \overline{\lambda^{n}} I_{m}\right) \geq 0 .
\end{aligned}
$$

Finally, it remains to consider the case where different $\lambda$ appear in the canonical decomposition. For this, it suffices to use the canonical representation of the pair $(A, H)$ and write $N$ as a sum of rational matrix-valued functions $N_{i}$ satisfying $N_{i}(z)^{*}=N_{i}(\bar{z})$ and each having a single pole.

The matrix $H$ is called the Hermitian matrix associated with a given realization. From (2.10) it follows that $H^{-1}$ is the Gram matrix of $\mathcal{L}(N)$ with respect to the basis under consideration; for $c, d \in \mathbb{C}^{m}$ we have

$$
\left\langle C\left(z I_{m}-A\right)^{-1} c, C\left(z I_{m}-A\right)^{-1} d\right\rangle_{\mathcal{L}(N)}=d^{*} H^{-1} c .
$$

If $H>0$, without loss of generality we may assume that $H=I_{m}$. This amounts to the replacement of $\left(\begin{array}{ll}A & B \\ C & D\end{array}\right)$ by

$$
\left(\begin{array}{cc}
\sqrt{H} & 0 \\
0 & I_{n}
\end{array}\right)\left(\begin{array}{ll}
A & B \\
C & D
\end{array}\right)\left(\begin{array}{cc}
\sqrt{H}^{-1} & 0 \\
0 & I_{n}
\end{array}\right) .
$$


Proposition 2.6. In the notation of the preceding proposition, the space $\mathcal{L}(N)$ consists of functions $F$ of the form

$$
F(z)=C\left(z I_{m}-A\right)^{-1} x, \quad x \in \mathbb{C}^{m},
$$

with the inner product

$$
\langle F, G\rangle_{\mathcal{L}(N)}=y^{*} H^{-1} x,
$$

where $G(z)=C\left(z I_{m}-A\right)^{-1} y$ with $y \in \mathbb{C}^{m}$. In particular, $\mathcal{L}(N)$ contains no nonzero constant functions.

Proof. Since the realization of $N$ is minimal, the pair $(C, A)$ is observable, that is,

$$
\bigcap_{k=0}^{\infty} \operatorname{ker} C A^{k}=\{0\}
$$

Hence,

$$
C\left(z I_{m}-A\right)^{-1} x \equiv 0 \Longrightarrow x=0,
$$

so that $F$ and the associated inner product (2.16) are well defined.

We take

$$
x=H\left(\sum_{j=1}^{k}\left(w_{j} I_{m}-A\right)^{-*} C^{*} e_{j}\right)
$$

and

$$
y=H\left(\sum_{j=1}^{k}\left(v_{j} I_{m}-A\right)^{-*} C^{*} d_{j}\right),
$$

where the $e_{j}$ and $d_{j}$ belong to $\mathbb{C}^{n}$. Then

$$
\begin{aligned}
& F(z)=C\left(z I_{m}-A\right)^{-1} x=\sum_{j=1}^{k} K_{N}\left(z, w_{j}\right) e_{j}, \\
& G(z)=C\left(z I_{m}-A\right)^{-1} x=\sum_{j=1}^{k} K_{N}\left(z, v_{j}\right) d_{j},
\end{aligned}
$$

and

$$
\langle F, G\rangle_{\mathcal{L}(N)}=\sum_{i, j=1}^{k} d_{i}^{*} K\left(v_{i}, w_{j}\right) e_{j}=y^{*} H^{-1} x .
$$

This finishes the proof of (2.16) because such $x, y$ span $\mathbb{C}^{m}$. Now we show that $\mathcal{L}(N)$ contains no nonzero constant functions. Assume that $x \in \mathbb{C}^{m}$ is such that $C\left(z I_{m}-A\right)^{-1} x$ is a constant function, say $t$. Multiplying the identity $C\left(z I_{m}-A\right)^{-1} x \equiv t$ by $z$ on both sides and letting $z \rightarrow \infty$, we see that $t=0$.

Now, we consider the case where $N$ is not necessarily analytic at infinity.

Proposition 2.7. The space $\mathcal{L}(N)$ is finite-dimensional if and only if $N$ is of the form

$$
N(z)=D+E z+\sum_{j=1}^{\ell} \frac{M_{j}}{t_{j}-z},
$$

where $D$ is a selfadjoint matrix, $E$ and $M_{j}$ are positive matrices, and the $t_{j}$ are real numbers. Furthermore, $\mathcal{L}(N)$ consists of functions $F$ of the form

$$
F(z)=E e+\sum_{j=1}^{\ell} \frac{M_{j} e_{j}}{t_{j}-z}, \quad e, e_{1}, \ldots, e_{\ell} \in \mathbb{C}^{n},
$$


with the inner product

$$
\langle F, G\rangle_{\mathcal{L}(N)}=d^{*} E e+\sum_{j=1}^{\ell} d_{j}^{*} M_{j} e_{j}
$$

where

$$
G(z)=E d+\sum_{j=1}^{\ell} \frac{M_{j} d_{j}}{t_{j}-z}, \quad d, d_{1}, \ldots, d_{\ell} \in \mathbb{C}^{n} .
$$

Proof. First, assume that $N$ is analytic at infinity; we take a minimal realization of $N$ with the associated Hermitian matrix $H$ equal to $I_{m}$. Then

$$
N(z)=D-C\left(z I_{m}-A\right)^{-1} C^{*},
$$

where $A=A^{*}$. Let $U$ be a unitary matrix such that

$$
A=U \operatorname{diag}\left(t_{1} I_{m_{1}}, \ldots, t_{\ell} I_{m_{\ell}}\right) U^{*},
$$

where the $t_{j}$ are real and are assumed to be pairwise different. Then

$$
N(z)=D-C U\left(z I_{m}-\operatorname{diag}\left(t_{1} I_{m_{1}}, \ldots, t_{\ell} I_{m_{\ell}}\right)\right)^{-1} U^{*} C^{*} .
$$

Let $C U=\left(c_{1} \cdots c_{\ell}\right)$. We arrive at (2.17) with $E=0$ and $M_{j}=c_{j} c_{j}^{*}$.

Now, assume that $N$ is not analytic at infinity. There exists a real number $t_{0}$ such that the function $M(z)=N\left(1 /\left(z-t_{0}\right)\right)$ is analytic at infinity. Since $M(z)=M(\bar{z})^{*}$, and the function $M(z)$ still has positive imaginary part in $\mathbb{C}_{+}$, we can write

$$
M(z)=D+\sum_{j=1}^{\ell} \frac{M_{j}}{t_{j}-z} .
$$

Thus,

$$
\begin{aligned}
N(z) & =M\left(\left(1+z t_{0}\right) / z\right) \\
& =D-\sum_{j=1}^{\ell} \frac{M_{j}}{t_{j}-\frac{1+z t_{0}}{z}} \\
& =D-\sum_{j=1}^{\ell} \frac{z M_{j}}{\left(t_{j}-t_{0}\right) z-1} .
\end{aligned}
$$

The map $\frac{1}{z-t_{0}}$ sends $t_{0}$ to $\infty$; therefore, one of the $t_{j}$ (say $t_{1}$ ) is equal to $t_{0}$, and we have

$$
\begin{aligned}
N(z) & =D+z M_{1}-\sum_{2}^{\ell} \frac{z M_{j}}{\left(t_{j}-t_{0}\right) z-1} \\
& =\left(D-\sum_{2}^{\ell} \frac{M_{j}}{t_{j}-t_{0}}\right)-\left(\sum_{2}^{\ell} \frac{M_{j}}{\left(t_{j}-t_{0}\right) z-1}-\frac{M_{j}}{t_{j}-t_{0}}\right) \\
& =D^{\prime}+\sum_{2}^{\ell} \frac{M_{j}^{\prime}}{t_{j}^{\prime}-z}
\end{aligned}
$$

with

$$
D^{\prime}=\left(D-\sum_{2}^{\ell} \frac{M_{j}}{t_{j}-t_{0}}\right) \text { and } M_{j}^{\prime}=\frac{M_{j}}{\left(t_{j}-t_{0}\right)^{2}} \quad \text { for } j=2, \ldots, \ell .
$$

Thus, $N$ is of the required form. 
Now we describe the space $\mathcal{L}(N)$ with $N$ as in (2.17). We have

$$
F(z)=\sum_{1}^{p} \frac{N(z)-N\left(w_{k}\right)^{*}}{z-\overline{w_{k}}} e_{k}=E\left(\sum_{1}^{p} e_{k}\right)+\sum_{1}^{\ell} \frac{M_{j}\left(\sum_{1}^{p} e_{k}\right)}{\left(t_{j}-t\right)(\bar{w}-t)}
$$

and

$$
G(z)=\sum_{1}^{p} \frac{N(z)-N\left(v_{k}\right)^{*}}{z-\overline{v_{k}}} d_{k}=E\left(\sum_{1}^{p} d_{k}\right)+\sum_{1}^{\ell} \frac{M_{j}\left(\sum_{1}^{p} d_{k}\right)}{\left(t_{j}-t\right)(\bar{w}-t)}
$$

whence

$$
\begin{aligned}
\langle F, G\rangle_{\mathcal{L}(N)}=\sum_{i, j=1}^{p} e_{j}^{*} \frac{N\left(v_{k}\right)-N\left(w_{j}\right)^{*}}{v_{k}-\overline{w_{j}}} d_{k} \\
=\left(\sum_{1}^{p} d_{k}\right)^{*} B\left(\sum_{1}^{p} c_{k}\right)+\sum_{1}^{\ell}\left(\sum_{1}^{p} d_{k}\right)^{*} M_{j}\left(\sum_{1}^{p} c_{k}\right) .
\end{aligned}
$$

Corollary 2.8. The space $\mathcal{L}(N)$ contains no nonzero constants if and only if $N$ is analytic at infinity.

Proof. This follows from (2.17) and (2.18).

It should be noted that the uniqueness of a reproducing kernel implies that $N_{1}$ and $N_{2}$ correspond to the same space if and only if

$$
N_{1}(z)=N_{2}(z)+D \text {, }
$$

where $D$ is a selfadjoint matrix. The role of $D$ will be important when the function $-N^{-1}$ is considered together with $N$.

Example 2.9. If $N_{1}(z)=-\frac{1}{z}$, from the relation

$$
\frac{N_{1}(z)-\overline{N_{1}(w)}}{z-\bar{w}}=\frac{1}{z \bar{w}}
$$

it follows that the space $\mathcal{L}(N)$ consists of the multiples of the function $\frac{1}{z}$ with the norm $\left\|\frac{1}{z}\right\|_{\mathcal{N}}=1$. The function $-1 / N_{1}(z)=z$ is not analytic at infinity, and the corresponding space $\mathcal{L}\left(-1 / N_{1}\right)$ consists of constants because

$$
\frac{-1 / N_{1}(z)-\left(\overline{-1 / N_{1}(w)}\right)}{z-\bar{w}}=\frac{z-\bar{w}}{z-\bar{w}}=1 .
$$

On the other hand, if we take $N_{2}(z)=1+N_{1}(z)$, then the space $\mathcal{L}(N)$ will not change, but $-N_{2}(z)^{-1}=\frac{z}{1-z}$ is analytic at infinity. We have

$$
\frac{N_{2}(z)-\overline{N_{2}(w)}}{z-\bar{w}}=\frac{1}{(1-z)(1-\bar{w})} .
$$

The space $\mathcal{L}\left(N_{2}\right)$ consists of the multiples of the function $\frac{1}{1-z}$ and contains no nonzero constants.

Another proof of Proposition 2.5 involves the Riesz-Herglotz representation formula for analytic functions with a positive imaginary part in the open upper half-plane. This proof is much shorter. We chose to present direct arguments based on the theory of realization of rational functions and on the theory of finite-dimensional reproducing-kernel Hilbert spaces. The case of finite-dimensional Pontryagin spaces with such reproducing kernels was considered in [3]. As in [3], we make use of the realization theory, but our approach and point of view are slightly different. 
2.3. Model for one matrix. In this section we use $\mathcal{L}(N)$-spaces to give models for selfadjoint matrices.

Theorem 2.10. Let $G_{+} \in \mathbb{C}^{m \times m}$ be a selfadjoint matrix, let $C \in \mathbb{C}^{n \times m}$ be such that

$$
\bigcap_{k=0}^{\infty} \operatorname{ker} C G_{+}^{k}=\{0\}
$$

and let $\mathcal{H}$ denote the Hilbert space of $\mathbb{C}^{n}$-valued functions of the form $F(z)=C\left(z I_{m}-\right.$ $\left.G_{+}\right)^{-1} x$ with $x \in \mathbb{C}^{m} ; \mathcal{H}$ is endowed with the inner product

$$
\langle F, G\rangle_{\mathcal{H}}=y^{*} x, \quad \text { where } G(z)=C\left(z I_{m}-G_{+}\right)^{-1} y, y \in \mathbb{C}^{m} .
$$

Then $\mathcal{H}$ is a reproducing-kernel Hilbert space, and its reproducing kernel is of the form

$$
\frac{N(z)-N(w)^{*}}{z-\bar{w}}
$$

where

$$
N(z)=D+C\left(G_{+}-z I_{m}\right)^{-1} C^{*},
$$

$D$ being any selfadjoint matrix in $\mathbb{C}^{n \times n}$.

Proof. From (2.19) we see that

$$
C\left(z I_{m}-G_{+}\right)^{-1} x \equiv 0 \Longrightarrow x=0,
$$

so that the inner product (2.20) is well defined. For $w \in \mathbb{C} \backslash \mathbb{R}$, set

$$
K(z, w)=C\left(z I_{m}-G_{+}\right)^{-1}\left(w I_{m}-G_{+}\right)^{-*} C^{*} .
$$

Then for every $w \in \mathbb{C} \backslash \mathbb{R}$ and every $y \in \mathbb{C}^{m}$ the function $z \mapsto K(z, w) y$ belongs to $\mathcal{H}$. Furthermore, for $F(z)=C\left(z I_{m}-G_{+}\right)^{-1} x \in \mathcal{H}$ we have

$$
\langle F(\cdot), K(\cdot, w) y\rangle_{\mathcal{H}}=\left(\left(w I_{m}-G_{+}\right)^{-*} C^{*} y\right)^{*} x=y^{*} C\left(w I_{m}-G_{+}\right)^{-1} x=y^{*} F(w) .
$$

Thus, the reproducing kernel of $\mathcal{H}$ is

$$
K(z, w)=C\left(z I_{m}-G_{+}\right)^{-1}\left(w I_{m}-G_{+}\right)^{-*} C^{*} .
$$

Finally, let $N$ be as in (2.21). Then

$$
\begin{gathered}
\frac{N(z)-N(w)^{*}}{z-\bar{w}}=\frac{C\left(z I_{m}-G_{+}\right)^{-1} C^{*}-C\left(w I_{m}-G_{+}\right)^{-*} C^{*}}{z-\bar{w}} \\
=C\left\{\frac{\left(z I_{m}-G_{+}\right)^{-1}-\left(\bar{w} I_{m}-G_{+}\right)^{-1}}{z-\bar{w}}\right\} \\
=C\left(z I_{m}-G_{+}\right)^{-1}\left(w I_{m}-G_{+}\right)^{-*} C^{*},
\end{gathered}
$$

which completes the proof in view of (2.22).

Proposition 2.11. Condition (2.19) is equivalent to the condition

$$
\bigcap_{z \in \mathbb{C}_{+}} \operatorname{ker} C\left(z I_{m}-G\right)^{-1}=\{0\} .
$$

Proof. This follows from the power series expansion at infinity:

$$
C\left(z I_{m}-G\right)^{-1}=\sum_{\ell=0}^{\infty} \frac{1}{z^{\ell+1}} C G^{\ell} .
$$


Theorem 2.12. Let $G_{+} \in \mathbb{C}^{m \times m}$ be a selfadjoint matrix, and let $C \in \mathbb{C}^{n \times m}$ be a matrix satisfying (2.19). Let $N$ be defined by (2.21), and let $\mathcal{L}(N)$ be the reproducing-kernel Hilbert space with reproducing kernel $\frac{N(z)-N(w)^{*}}{z-\bar{w}}$. Then the map $U_{+}$defined by

$$
U_{+} x(z)=C\left(z I_{m}-G_{+}\right)^{-1} x
$$

is a unitary map from $\mathbb{C}^{m}$ (endowed with the canonical inner product) onto $\mathcal{L}(N)$. Its adjoint is given by

$$
U_{+}^{*}\left(C\left(z I_{m}-G_{+}\right)^{-1} y\right)=y
$$

Finally, we have

$$
\begin{aligned}
U_{+} G_{+} U_{+}^{*} F(z) & =z F(z)+c_{F}, \\
U_{+}\left(G_{+}-\alpha I_{m}\right)^{-1} U_{+}^{*} F(z) & =R_{\alpha} F(z),
\end{aligned}
$$

where $c_{F}=-\lim _{z \rightarrow \infty} z F(z)$.

Proof. First, we compute $U_{+}^{*}$. Let $e \in \mathbb{C}^{n}$, and let $w \in \mathbb{C} \backslash \mathbb{R}$. We have

$$
\begin{aligned}
\left\langle U_{+}^{*}\right. & \left.\left(C\left(z I_{m}-G_{+}\right)^{-1} y\right),\left(\bar{w} I_{m}-G_{+}\right)^{-1} C^{*} e\right\rangle_{\mathbb{C}^{m}} \\
& =\left\langle C\left(z I_{m}-G_{+}\right)^{-1} y, U_{+}\left(\bar{w} I_{m}-G_{+}\right)^{-1} C^{*} e\right\rangle_{\mathcal{L}(N)} \\
& =\left\langle C\left(z I_{m}-G_{+}\right)^{-1} y, C\left(z I_{m}-G_{+}\right)^{-1}\left(\bar{w} I_{m}-G_{+}\right)^{-1} C^{*} e\right\rangle_{\mathcal{L}(N)} \\
& =\left\langle\left(C\left(z I_{m}-G_{+}\right)^{-1} y\right), K_{N}(z, w) e\right\rangle_{\mathcal{L}(N)} \\
& =e^{*} C\left(w I_{m}-G_{+}\right)^{-1} y
\end{aligned}
$$

where we have used (2.30) and the reproducing-kernel property. It follows that

$$
U_{+}^{*}\left(C\left(z I_{m}-G_{+}\right)^{-1} y\right)=y
$$

because the span of the vectors $\left(\bar{w} I_{m}-G_{+}\right)^{-1} C^{*} e$ with $w \in \mathbb{C} \backslash \mathbb{R}$ and $e \in \mathbb{C}^{n}$ is equal to $\mathbb{C}^{m}$.

To prove the next claim, we write

$$
\begin{gathered}
C\left(z I_{m}-G_{+}\right)^{-1} G_{+} x=C\left(z I_{m}-G_{+}\right)^{-1}\left(G_{+}-z I_{m}+z I_{m}\right) x \\
\quad=z C\left(z I_{m}-G_{+}\right)^{-1} x-C x
\end{gathered}
$$

and observe that

$$
C x=\lim _{z \rightarrow \infty} z C\left(z I_{m}-G_{+}\right)^{-1} x=-\lim _{z \rightarrow \infty} z F(z) .
$$

The last claim is proved as follows. Let $F(z)=C\left(z I_{m}-G_{+}\right)^{-1} x$ for some $x \in \mathbb{C}^{m}$. Then

$$
\begin{aligned}
R_{\alpha} F(z) & =C \frac{\left(z I_{m}-G_{+}\right)^{-1}-\left(\alpha I_{m}-G_{+}\right)^{-1}}{z-\alpha} x \\
& =C\left(z I_{m}-G_{+}\right)^{-1}\left(G_{+}-\alpha I_{m}\right)^{-1} x .
\end{aligned}
$$

2.4. The model of de Branges and Rovnyak for pairs of matrices. In this subsection we discuss the model of de Branges and Rovnyak for pairs of selfadjoint matrices. More generally, the model for pairs of bounded selfadjoint operators will be considered in Subsection 4.2, and the model in the setting of possibly unbounded operators will be given in $\$ 5$

Our starting point is a pair of selfadjoint matrices $G_{+}$and $G_{-}$in $\mathbb{C}^{m \times m}$. We assume that $G_{+}$and $G_{-}$do not coincide on any nontrivial subspace of $\mathbb{C}^{r}$ invariant under both $G_{+}$and $G_{-}$. We set

$$
n=\operatorname{dim} \operatorname{ran}\left(G_{+}-G_{-}\right)
$$


D. ALPAY AND I. GOHBERG

and write $G_{+}-G_{-}=-C^{*} J C$, where $J \in \mathbb{C}^{n \times n}$ is both selfadjoint and unitary and $C \in \mathbb{C}^{n \times r}$. The function

$$
N(z)=J+C\left(G_{+}-z I\right)^{-1} C^{*}
$$

is called the characteristic function of the pair. The function $N$ allows us to recover the pair $\left(G_{+}, G_{-}\right)$up to a unitary similarity.

The function $N$ satisfies $N(\bar{z})^{*}=N(z)$ and has positive imaginary part; in particular, the function $K_{N}(z, w)$ is positive for $z, w \in \mathbb{C}$ that are not poles of $N$.

Proposition 2.13. Let $G_{+}$and $G_{-}$be two selfadjoint matrices in $\mathbb{C}^{m \times m}$, and let

$$
\mathcal{E}=\bigcap_{k=0}^{\infty} \operatorname{ker}\left(G_{+}^{k}-G_{-}^{k}\right)
$$

Then $\mathcal{E}$ is the largest linear subspace invariant under both $G_{+}$and $G_{-}$and on which $G_{+}=G_{-}$.

Proof. The matrices $G_{+}$and $G_{-}$coincide on $\mathcal{E}$ since $\mathcal{E} \subset \operatorname{ker}\left(G_{+}-G_{-}\right)$. Furthermore, $\mathcal{E}$ is invariant under the operators $G_{+}$and $G_{-}$. Indeed, let $u \in \mathcal{E}$. We want to show that $G_{ \pm} u \in \mathcal{E}$, that is,

$$
G_{+}^{k}\left(G_{ \pm} u\right)=G_{-}^{k}\left(G_{ \pm} u\right) \text { for all } k .
$$

This is readily seen because $G_{+} u=G_{-} u$.

Let $\mathcal{M}$ be such that $G_{ \pm} \mathcal{M} \subset \mathcal{M}$, and let $u \in \mathcal{M}$. Then $G_{+} u=G_{-} u$. By assumption, both $G_{+} u$ and $G_{-} u$ also belong to $\mathcal{M}$, whence

$$
G_{+}\left(G_{+} u\right)=G_{-}\left(G_{+} u\right) .
$$

Since $G_{+} u=G_{-} u$, we obtain $G_{+}^{2} u=G_{-}^{2} u$. By induction, we conclude that $G_{+}^{k} u=G_{-}^{k} u$ for all $k$, and therefore $\mathcal{M} \subset \mathcal{E}$.

Thus, the condition

$$
\mathcal{E}=\bigcap_{k=0}^{\infty} \operatorname{ker}\left(G_{+}^{k}-G_{-}^{k}\right)=\{0\}
$$

is necessary and sufficient for the matrices $G_{+}$and $G_{-}$to have no nontrivial invariant subspace on which they coincide. Another characterization of the fact that $\mathcal{E}=\{0\}$ is as follows.

Lemma 2.14. Two selfadjoint matrices $G_{+}$and $G_{-}$in $\mathbb{C}^{m \times m}$ satisfy condition (2.26) if and only if they have no common eigenvalue corresponding to the same eigenvector.

Proof. Since $\mathcal{E}$ is $G_{+}$-invariant, there exists a nonzero vector $u \in \mathbb{C}^{m}$ and a real number $\lambda$ such that $G_{+} u=\lambda u$. In particular, since $u \in \mathcal{E}$, we have $G_{+} u=G_{-} u$, whence $G_{-} u=\lambda u$. Thus, $G_{+}$and $G_{-}$have a common eigenvector with the same eigenvalue. The converse statement is clear.

As was proved in Proposition 2.6 the space $\mathcal{L}(N)$ in Theorem 2.12] contains no nonzero constants. If $\operatorname{det} D \neq 0$, we can consider the function $-N(z)^{-1}$ and the corresponding space $\mathcal{L}\left(-N^{-1}\right)$. It contains no nonzero constants, and the operators $R_{\alpha}$ in $\mathcal{L}\left(-N^{-1}\right)$ are the resolvents of a Hermitian matrix. So, then there are two Hermitian matrices associated with $N$ in a natural way. This is the idea behind the result of de Branges and Rovnyak that gives a model for a pair of selfadjoint operators in terms of a single Herglotz-Nevanlinna function. In the next theorem we construct the HerglotzNevanlinna function associated with a pair of matrices. 
Theorem 2.15. Let $G_{+}$and $G_{-}$be two selfadjoint matrices in $\mathbb{C}^{m \times m}$ such that (2.26) is true. Let $n=\operatorname{rank}\left(G_{+}-G_{-}\right)$, and let

$$
G_{+}-G_{-}=-C^{*} J C,
$$

where $J \in \mathbb{C}^{n \times n}$ is both selfadjoint and unitary, and $C \in \mathbb{C}^{n \times m}$. Let $N$ be defined by the formula

$$
N(z)=J+C\left(G_{+}-z I_{m}\right)^{-1} C^{*} .
$$

Then $N$ has a positive imaginary part in the open upper half-plane and satisfies $N(z)=$ $N(\bar{z})^{*}$. Moreover,

$$
-N(z)^{-1}=-J+J C\left(G_{-}-z I_{m}\right)^{-1} C^{*} J,
$$

and (2.28) and (2.29) are minimal realizations of $N$ and $-N^{-1}$, respectively.

Proof. The claim about $N$ follows from the identity

$$
\frac{N(z)-N(w)^{*}}{z-\bar{w}}=C\left(G_{+}-z I_{m}\right)^{-1}\left(G_{+}-\bar{w} I_{m}\right)^{-1} C^{*} .
$$

First, we prove that the pair $\left(C, G_{+}\right)$is observable, i.e., $\bigcap_{k=0}^{\infty} \operatorname{ker} C G_{+}^{k}=\{0\}$. Let $u \in \bigcap_{k=0}^{\infty} \operatorname{ker} C G_{+}^{k}$. In particular, $C u=0$, whence $C^{*} J C u=0$, that is, $G_{+} u=$ $G_{-} u$. Similarly, from the condition $C G_{+} u=0$ we obtain $C^{*} J C G_{+} u=0$, and thus $\left(G_{+}-G_{-}\right) G_{+} u=0$. It follows that $G_{+}^{2} u=G_{-}^{2} u$, since we already know that $G_{+} u=$ $G_{-} u$. By induction, in a similar way we prove that $G_{+}^{k} u=G_{-}^{k} u$. Thus, $u \in$ $\bigcap_{k=0}^{\infty} \operatorname{ker}\left(G_{+}^{k}-G_{-}^{k}\right)$, and we see that $u=0$. Therefore, the pair $\left(C, G_{+}\right)$is observable; consequently, the pair $\left(G_{+}, C^{*}\right)$ is controllable, which implies that the realization $(2.28)$ is minimal.

As a consequence we obtain a description of the spaces $\mathcal{L}(N)$ and $\mathcal{L}\left(-N^{-1}\right)$. The infinite-dimensional counterpart of this result is presented in Theorem 3.2

Corollary 2.16. We have

$$
\mathcal{L}(N)=\left\{F(z)=C\left(z I_{m}-G_{+}\right)^{-1} x, x \in \mathbb{C}^{n}\right\}
$$

and

$$
\mathcal{L}\left(-N^{-1}\right)=\left\{F(z)=J C\left(z I_{m}-G_{-}\right)^{-1} x, x \in \mathbb{C}^{n}\right\},
$$

with the inner products

$$
\langle F, G\rangle_{\mathcal{L}(N)}=y^{*} x, \quad \text { where } G(z)=C\left(z I_{m}-G_{+}\right)^{-1} y,
$$

and

$$
\langle F, G\rangle_{\mathcal{L}\left(-N^{-1}\right)}=y^{*} x, \quad \text { where } G(z)=J C\left(z I_{m}-G_{-}\right)^{-1} y,
$$

respectively. Finally, the map $F \mapsto-N^{-1} F$ is a unitary map from $\mathcal{L}(N)$ to $\mathcal{L}\left(-N^{-1}\right)$.

Proof. The claim about $\mathcal{L}(N)$ follows from Proposition 2.6 The claim about $\mathcal{L}\left(-N^{-1}\right)$ follows from (2.29) and the relation

$$
\frac{-N(z)^{-1}+N(w)^{-*}}{z-\bar{w}}=J C\left(G_{-}-z I_{m}\right)^{-1}\left(G_{-}-\bar{w} I_{m}\right)^{-1} C^{*} J .
$$


Now we prove the last claim. Let $F \in \mathcal{L}(N)$, i.e., $F(z)=C\left(z I_{m}-G_{+}\right)^{-1} x$ for some $x \in \mathbb{C}^{m}$. We have

$$
\begin{aligned}
&-N(z)^{-1} F(z)=\left(-J+J C\left(G_{-}-z I_{m}\right)^{-1} C^{*} J\right) C\left(z I_{m}-G_{+}\right)^{-1} x \\
&=-J C\left(z I_{m}-G_{+}\right)^{-1} x+J C\left(G_{-}-z I_{m}\right)^{-1}\left(G_{-}-G_{+}\right)\left(z I_{m}-G_{+}\right)^{-1} x \\
&=-J C\left(z I_{m}-G_{+}\right)^{-1} x \\
&+J C\left(G_{-}-z I_{m}\right)^{-1}\left(G_{-}-z I_{m}+z I_{m}-G_{+}\right)\left(z I_{m}-G_{+}\right)^{-1} x \\
&= J C\left(G_{-}-z I_{m}\right)^{-1} x .
\end{aligned}
$$

Thus, $-N^{-1} F$ belongs to $\mathcal{L}\left(-N^{-1}\right)$ and has the same norm as $F$ :

$$
\left\|-N^{-1} F\right\|_{\mathcal{L}\left(-N^{-1}\right)}^{2}=\|F\|_{\mathcal{L}(N)}^{2}=x^{*} x .
$$

The role of the characteristic function of the pair $\left(G_{+}, G_{-}\right)$of matrices is clarified in the next theorem, which is the main result of the section. The passage from $N$ to $-N^{-1}$ can be viewed in two equivalent ways: one can look at $G_{+}$and $G_{-}$in $\mathcal{L}(N)$ and view $G_{-}$as a perturbation of $G_{+}$. One can also look at both $G_{+}$and $G_{-}$in the space $\mathcal{L}\left(-N^{-1}\right)$ and view $G_{+}$as a perturbation of $G_{-}$. See also the next section and de Branges' paper [18, p. 546], where such an interpretation is given in terms of measures for the infinite-dimensional case.

Theorem 2.17. For the map $U_{+}$, we have

$$
\begin{aligned}
U_{+} G_{+} U_{+}^{*} F(z) & =z U_{+} F(z)+c_{F}, \\
U_{+} G_{-} U_{+}^{*} F(z) & =z F(z)+N(z) J c_{F}, \\
U_{+}\left(G_{+}-\alpha I_{m}\right)^{-1} U_{+}^{*} F & =R_{\alpha} F, \\
U_{+}\left(G_{-}-\alpha I_{m}\right)^{-1} U_{+}^{*} F & =R_{\alpha} F-\left(R_{\alpha} N\right) N(\alpha)^{-1} F(\alpha),
\end{aligned}
$$

where $F \in \mathcal{L}(N)$ and

$$
c_{F}=-\lim _{z \rightarrow \infty} z F(z)
$$

In particular,

$$
U_{+}\left\{\left(G_{+}-\alpha I_{m}\right)^{-1}-\left(G_{-}-\alpha I_{m}\right)^{-1}\right\} U_{+}^{*} F=R_{\alpha} N N(\alpha)^{-1} F(\alpha) .
$$

Similarly, the map $U_{-}$that takes $u \in \mathbb{C}^{m}$ to the rational function $z \mapsto J C\left(z I-G_{-}\right)^{-1} u$ is an isometry from the space $\mathbb{C}^{m}$ endowed with its usual inner product onto $\mathcal{L}\left(-N^{-1}\right)$. We have $\mathcal{M}_{-N^{-1}} U_{+}=U_{-}$. Moreover, for $U_{-}$we have

$$
\begin{aligned}
U_{-} G_{-} U_{-}^{*} F(z) & =z U_{-} F(z)+c_{F}, \\
U_{-} G_{+} U_{-}^{*} F(z) & =z F(z)+N^{-1}(z) J c_{F}, \\
U_{-}\left(G_{-}-\alpha I_{m}\right)^{-1} U_{-}^{*} F & =R_{\alpha} F \\
U_{-}\left(G_{+}-\alpha I_{m}\right)^{-1} U_{-}^{*} F & =R_{\alpha} F-\left(R_{\alpha} N^{-1}\right) N(\alpha) F(\alpha), \quad F \in \mathcal{L}\left(-N^{-1}\right) .
\end{aligned}
$$

In particular,

$$
\begin{gathered}
U_{-}\left\{\left(G_{+}-\alpha I_{m}\right)^{-1}-\left(G_{-}-\alpha I_{m}\right)^{-1}\right\} U_{-}^{*} F \\
=-R_{\alpha} N^{-1} N(\alpha) F(\alpha), \quad F \in \mathcal{L}\left(-N^{-1}\right) .
\end{gathered}
$$


Proof. Let $F \in \mathcal{L}(N)$. By Corollary 2.16 $F(z)=C\left(z I_{m}-G_{+}\right)^{-1} x$ for a unique $x \in \mathbb{C}^{m}$. By Lemma 2.12 $U_{+}^{*} F=x$, and therefore

$$
\begin{aligned}
& U_{+} G_{+} U_{+}^{*} F(z)=C\left(z I_{m}-G_{+}\right)^{-1} G_{+} x \\
& \quad=C\left(z I_{m}-G_{+}\right)^{-1}\left(G_{+}-z I_{m}+z I_{m}\right) x \\
& \quad=z F(z)-C x,
\end{aligned}
$$

which completes the proof of (2.31) since, as was already noticed, $C x=-\lim _{z \rightarrow \infty} z F(z)$.

We turn to (2.32). Now we have

$$
\begin{aligned}
U_{+} G_{-} & U_{+}^{*} F(z)=C\left(z I_{m}-G_{+}\right)^{-1} G_{-} x \\
& =C\left(z I_{m}-G_{+}\right)^{-1}\left(G_{+}+G_{-}-G_{+}\right) x \\
& =C\left(z I_{m}-G_{+}\right)^{-1} G_{+} x+C\left(z I_{m}-G_{+}\right)^{-1} C^{*} J C \\
& =C\left(z I_{m}-G_{+}\right)^{-1} G_{+} x+C\left(z I_{m}-G_{+}\right)^{-1} C^{*} J C x \\
& =z F(z)-C x+(J-N(z)) J C x,
\end{aligned}
$$

where we have used (2.31) and (2.27), and we obtain

$$
U_{+} G_{-} U_{+}^{*} F(z)=z F(z)-N(z) J C x .
$$

To prove 2.33), we proceed as follows:

$$
\begin{aligned}
& U_{+}\left(\left(G_{+}-\alpha I_{m}\right)^{-1}\right) U_{+}^{*} F(z)=C\left(z I_{m}-G_{+}\right)^{-1}\left(G_{+}-\alpha I_{m}\right)^{-1} y \\
& \quad=\frac{C\left(z I_{m}-G_{+}\right)^{-1}-C\left(\alpha I_{m}-G_{+}\right)^{-1}}{z-\alpha} \\
& \quad=R_{\alpha} F(z) .
\end{aligned}
$$

For the proof of (2.34), we write

$$
\begin{aligned}
U_{+}\left(G_{-}\right. & \left.-\alpha I_{m}\right)^{-1} U_{+}^{*} F(z) \\
= & C\left(z I_{m}-G_{+}\right)^{-1}\left(G_{-}-\alpha I_{m}\right)^{-1} x \\
= & C\left(z I_{m}-G_{+}\right)^{-1}\left\{\left(G_{+}-\alpha I_{m}\right)^{-1}+\left(G_{-}-\alpha I_{m}\right)^{-1}-\left(G_{+}-\alpha I_{m}\right)^{-1}\right\} x \\
= & C\left(z I_{m}-G_{+}\right)^{-1}\left(G_{+}-\alpha I_{m}\right)^{-1} x \\
& +C\left(z I_{m}-G_{+}\right)^{-1}\left(G_{+}-\alpha I_{m}\right)^{-1}\left(G_{+}-G_{-}\right)\left(G_{-}-\alpha I_{m}\right)^{-1} x \\
= & C\left(z I_{m}-G_{+}\right)^{-1}\left(G_{+}-\alpha I_{m}\right)^{-1} x \\
& +C\left(z I_{m}-G_{+}\right)^{-1}\left(\alpha I_{m}-G_{+}\right)^{-1} C^{*} J C\left(G_{-}-\alpha I_{m}\right)^{-1} x .
\end{aligned}
$$

Using (2.33) and (2.30), we obtain

$$
U_{+}\left(G_{-}-\alpha I_{m}\right)^{-1} U_{+}^{*} F(z)=\frac{F(z)-F(\alpha)}{z-\alpha}+\frac{N(z)-N(\alpha)}{z-\alpha} J C\left(G_{-}-\alpha I_{m}\right)^{-1} x .
$$

To conclude the proof of (2.34), we observe that

$$
\begin{aligned}
-N(\alpha)^{-1} F(\alpha)= & -J C\left(\alpha I_{m}-G_{+}\right)^{-1} x+J C\left(G_{-}-\alpha I_{m}\right)^{-1} C^{*} J C\left(\alpha I_{m}-G_{+}\right)^{-1} x \\
= & -J C\left(\alpha I_{m}-G_{+}\right)^{-1} x \\
& +J C\left(G_{-}-\alpha I_{m}\right)^{-1}\left(G_{-}-\alpha I_{m}+\alpha I_{m}-G_{+}\right)\left(\alpha I_{m}-G_{+}\right)^{-1} x \\
= & J C\left(G_{-}-\alpha I_{m}\right)^{-1} x .
\end{aligned}
$$

This computation also shows that $\mathcal{M}_{-N^{-1}} U_{+}=U_{-}$.

The claims about $U_{-}$are proved in a similar way. 
It should be noted that, if $S$ is a unitary matrix and $K_{+}=S^{*} G_{+} S, K_{-}=S^{*} G_{-} S$, then

$$
K_{+}-K_{-}=S^{*} C^{*} J C S,
$$

and the characteristic function of the pair $\left(K_{+}, K_{-}\right)$is equal to

$$
J+C S\left(K_{+}-z I\right)^{-1} S^{*} C^{*}=J+C\left(G_{+}-z I\right)^{-1} C^{*},
$$

so that it is the same as the characteristic function of the pair $\left(G_{+}, G_{-}\right)$.

Similarly, the matrix $C$ in (2.27) is defined up to a left-multiplicative $J$-unitary constant. If we replace $C$ by $U C$, where $U J U^{*}=J$, then the characteristic function of the pair becomes

$$
J+U C\left(G_{+}-z I\right)^{-1} C^{*} U^{*}=U\left(J+C\left(G_{+}-z I\right)^{-1} C^{*}\right) U^{*}=U N(z) U^{*} .
$$

2.5. Trace formula for pairs of selfadjoint matrices. In this section we prove a trace formula and establish the relationship with Kreŭn's trace formula.

Proposition 2.18. Let $G_{+}$and $G_{-}$constitute a pair of selfadjoint matrices such that $\bigcap_{k=0}^{\infty} \operatorname{ker}\left(G_{+}^{k}-G_{-}^{k}\right)=\{0\}$, and let $N$ be the corresponding characteristic function. Put

$$
Y_{+}(w)=C\left(G_{+}-w I_{m}\right)^{-1} \quad \text { and } \quad Y_{-}(w)=\left(G_{-}-w I_{m}\right)^{-1} C^{*} J .
$$

Then for all $w \in \mathbb{C} \backslash \mathbb{R}$ we have

$$
\begin{aligned}
& N^{\prime}(w) N(w)^{-1}=Y_{+}(w) Y_{-}(w), \\
& \operatorname{Tr}\left\{\left(G_{+}-w I_{m}\right)^{-1}-\left(G_{-}-w I_{m}\right)^{-1}\right\}=\operatorname{Tr}\left(N^{\prime}(w) N(w)^{-1}\right), \\
& \quad \operatorname{det} N(w)=(\operatorname{det} J) \frac{\operatorname{det}\left(G_{+}-z I_{m}\right)}{\operatorname{det}\left(G_{-}-z I_{m}\right)} .
\end{aligned}
$$

Proof. We have $N^{\prime}(w)=C\left(G_{+}-w I\right)^{-2} C^{*}$, whence

$$
\begin{aligned}
& N^{\prime}(w) N(w)^{-1}=C\left(G_{+}-w I_{m}\right)^{-2} C^{*}\left(J-J C\left(G_{-}-w I_{m}\right)^{-1} C^{*} J\right) \\
&=C\left(G_{+}-w I_{m}\right)^{-2} C^{*} J-C\left(G_{+}-w I_{m}\right)^{-2} C^{*} J C\left(G_{-}-w I_{m}\right)^{-1} C^{*} J \\
&=C\left(G_{+}-w I_{m}\right)^{-2} C^{*} J-C\left(G_{+}-w I_{m}\right)^{-2}\left(G_{-}-G_{+}\right)\left(G_{-}-w I_{m}\right)^{-1} C^{*} J \\
&=C\left(G_{+}-w I_{m}\right)^{-2} C^{*} J \\
&-C\left(G_{+}-w I_{m}\right)^{-2}\left(G_{-}-w I_{m}+w I_{m}-G_{+}\right)\left(G_{-}-w I_{m}\right)^{-1} C^{*} J .
\end{aligned}
$$

Consequently,

$$
N^{\prime}(w) N(w)^{-1}=C\left(G_{+}-w I\right)^{-1}\left(G_{-}-w I\right)^{-1} C^{*} J
$$

and

$$
\begin{aligned}
& \operatorname{Tr} N^{\prime}(w) N(w)^{-1}=\operatorname{Tr}\left(G_{-}-w I_{m}\right)^{-1}\left(C^{*} J C\right)\left(G_{+}-w I_{m}\right)^{-1} \\
& \quad=\operatorname{Tr}\left(G_{-}-w I_{m}\right)^{-1}\left(G_{-}-w I+w I-G_{+}\right)\left(G_{+}-w I_{m}\right)^{-1} \\
& \quad=\operatorname{Tr}\left\{\left(G_{+}-w I_{m}\right)^{-1}-\left(G_{-}-w I_{m}\right)^{-1}\right\} .
\end{aligned}
$$

The last claim can be proved in two ways. First, we write

$$
\begin{aligned}
\operatorname{det} & N(w)=(\operatorname{det} J)\left(\operatorname{det}\left(I_{n}+J C\left(G_{+}-z I_{m}\right)^{-1} C^{*}\right)\right) \\
& \left.=(\operatorname{det} J)\left(\operatorname{det}\left(I_{n}+\left(G_{+}-z I_{m}\right)^{-1} C^{*} J C\right)\right)\right) \\
& =(\operatorname{det} J)\left(\operatorname{det}\left(I_{n}+\left(G_{+}-z I_{m}\right)^{-1}\left(G_{-}-G_{+}\right)\right)\right) \\
& =(\operatorname{det} J)\left(\operatorname{det}\left(G_{+}-z I_{m}\right)^{-1}\left(G_{-}-z I_{m}\right)\right) .
\end{aligned}
$$

Alternatively, we can use the formula (see [32, p. 129])

$$
\frac{d}{d w} \ln \operatorname{det} a(w)=\operatorname{Tr}\left(a^{\prime}(w) a^{-1}(w)\right)
$$


with $a(w)=N(w)$ and $a(w)=G_{ \pm}-w I_{m}$, and integrate (2.36).

In the scalar case we have the following uniqueness result.

Corollary 2.19. The characteristic function $N$ is a unique (up to a multiplicative constant) function analytic in $\mathbb{C}_{+}$and such that $(2.36)$ is true.

Proof. Indeed, two such functions, say $N_{1}$ and $N_{2}$, will satisfy

$$
\frac{N_{1}(w)^{\prime}}{N_{1}(w)}=\frac{N_{2}(w)^{\prime}}{N_{2}(w)}
$$

It follows that $\frac{N_{1}(w)}{N_{2}(w)}$ is constant, whence the result.

The next proposition remains true in the case of bounded operators, as we shall see in the sequel; to the best of our knowledge, it is new.

Proposition 2.20. Let $\gamma$ be a simple closed contour that contains the spectra of $G_{+}$and $G_{-}$, and let $f$ be a scalar-valued function analytic inside and on the boundary of $\gamma$. Then

$$
\operatorname{Tr}\left\{f\left(G_{-}\right)-f\left(G_{+}\right)\right\}=\frac{1}{2 \pi i} \int_{\gamma} \operatorname{Tr} N^{-1}(w)(N f)^{\prime}(w) d w
$$

Proof. From (2.37) we deduce that

$$
\operatorname{Tr}\left\{f(w)\left(G_{+}-w I_{m}\right)^{-1}-f(w)\left(G_{-}-w I_{m}\right)^{-1}\right\}=\operatorname{Tr} N^{\prime}(w) N^{-1}(w) f(w) .
$$

Integrating over the contour $\gamma$ and using the functional calculus, we obtain

$$
\operatorname{Tr}\left\{f\left(G_{-}\right)-f\left(G_{+}\right)\right\}=\frac{1}{2 \pi i} \int_{\gamma} \operatorname{Tr} N^{\prime}(w) N^{-1}(w) f(w) d w .
$$

We note that

$$
N^{-1}(w)(N f)^{\prime}(w)=f^{\prime}(w)+N^{-1}(w) N^{\prime}(w) f(w) .
$$

Substituting in (2.41), we obtain

$$
\operatorname{Tr}\left\{f\left(G_{-}\right)-f\left(G_{+}\right)\right\}=\frac{1}{2 \pi i} \int_{\gamma} \operatorname{Tr}\left(N^{-1}(w)(N f)^{\prime}(w)-f(w)\right) d w
$$

Since $f$ is analytic on the boundary of $\gamma$, we have $\int_{\gamma} f^{\prime}(w)=0$, and this leads to (2.39).

Neither equation (2.40) nor equation (2.39) is Kreŭn's formula. To indicate a link with Kreı̆n's formula, we need the following statement.

Proposition 2.21. Let $N$ be the characteristic function of a pair of Hermitian matrices. Then there exists a function $\zeta(t)$ such that

$$
\operatorname{Tr} N^{\prime}(w) N^{-1}(w)=\int_{\mathbb{R}} \frac{\zeta(t) d t}{(t-w)^{2}}
$$

Proof. Formula (2.36) shows that $\operatorname{Tr} N^{\prime}(w) N^{-1}(w)$ can be written as a finite sum of terms of the form $\frac{n_{i}}{t_{i}-w}$, where $n_{i} \in \mathbb{Z}$ and the $t_{i}$ are real numbers. As in [32, p. 109], we 
arrange the $t_{i}$ in increasing order. We have

$$
\begin{gathered}
\sum_{i=1}^{M} \frac{n_{i}}{t_{i}-w}=-\sum_{i=1}^{M} n_{i} \int_{-\infty}^{t_{i}} \frac{d t}{(t-w)^{2}} \\
=-\sum_{i=1}^{M} \int_{\mathbb{R}} \frac{1_{\left(-\infty, t_{i}\right]} n_{i}}{(t-w)^{2}} d t \\
=-\int_{\mathbb{R}} \frac{\sum_{i=1}^{M} 1_{\left(-\infty, t_{i}\right]} n_{i}}{(t-w)^{2}} d t
\end{gathered}
$$

which has the required form with

$$
\zeta(t)=\sum_{i=1}^{M} 1_{\left(-\infty, t_{i}\right]} n_{i} .
$$

This is a particular case of [38, (0.8), p. 156].

For the case of the general Herglotz-Nevanlinna function, see the discussion in Subsection 4.3

Now we explain how (2.42) leads, via (2.40), to Kreŭn's formula. Using (2.42), we obtain

$$
\begin{aligned}
\operatorname{Tr}\{ & \left.f\left(G_{-}\right)-f\left(G_{+}\right)\right\}=\frac{1}{2 \pi i} \int_{\gamma} \operatorname{Tr} N^{\prime}(w) N^{-1}(w) f(w) d w \\
& =\frac{1}{2 \pi i} \int_{\gamma}\left(\int_{\mathbb{R}} \frac{\zeta(t) d t}{(t-w)^{2}}\right) f(w) d w \\
& =\int_{\mathbb{R}} \zeta(t)\left(\frac{1}{2 \pi i} \int_{\gamma} \frac{f(w)}{(t-w)^{2}} d w\right) \\
& =\int_{\mathbb{R}} \zeta(t) f^{\prime}(t) d t .
\end{aligned}
$$

2.6. Inverse problems. Let $N$ be the characteristic function of a pair $\left(G_{+}, G_{-}\right)$. Then, as we saw in the proof of Lemma 2.16, the spaces $\mathcal{L}(N)$ and $\mathcal{L}\left(-N^{-1}\right)$ contain no nonzero constant functions. It turns out that these conditions are also sufficient for a rational function with positive imaginary part in $\mathbb{C}_{+}$to be the characteristic function of a pair $\left(G_{+}, G_{-}\right)$, and that these conditions are easily restated in terms of a minimal realization of $N$.

Theorem 2.22. $A \mathbb{C}^{n \times n}$-valued rational function $N$ is the characteristic function of a pair $\left(G_{+}, G_{-}\right)$of matrices if and only if the following conditions are satisfied:

1) $N$ has positive imaginary part in $\mathbb{C}^{+}$;

2) $N(\bar{z})^{*}=N(z)$ for $z \in \mathbb{C} \backslash \mathbb{R}$;

3) $N$ is analytic at infinity and $N(\infty)$ is both selfadjoint and unitary.

If these conditions are fulfilled, then $N$ is the characteristic function of a pair of matrices. One such pair $\left(G_{+}, G_{-}\right)$can be constructed as follows: let $N(z)=D+C\left(A-z I_{m}\right)^{-1} C^{*}$ be a minimal realization of $N$ with the associated matrix equal to the identity. Then

$$
G_{+}=A \text { and } G_{-}=A-C^{*} D C .
$$

Any other pair is obtained from this pair by a unitary similarity.

Proof. Formula (2.28) shows that the above conditions are necessary. Conversely, if they are satisfied, then, by Proposition 2.5. $N$ admits a minimal realization of the form announced above with $H=I$. The pair $\left(G_{+}, G_{-}\right)$of operators is then built by using 
Theorem 2.17. We turn to the uniqueness claim. The realization of $N$ is unique up to a similarity operator $S$ : if

$$
N(z)=D+C_{1}\left(z I_{m}-A_{1}\right)^{-1} C_{1}^{*}
$$

is another minimal realization, then

$$
C_{1}=C S \quad \text { and } \quad A_{1}=S^{-1} A S .
$$

If, moreover, $A_{1}=A_{1}^{*}$, we see that $S^{-*}$ also satisfies these equations; hence, by the uniqueness of the similarity matrix $S=S^{-*}$, the operator $S$ is unitary, and the pairs $\left(A, A-C^{*} D C\right)$ and $\left(A_{1}, A_{1}-C_{1}^{*} D C_{1}\right)$ are related by

$$
A_{1}=S^{*} A S \quad \text { and } \quad A_{1}-C_{1}^{*} D C_{1}=S\left(A-C^{*} D C\right) S .
$$

We note that if $N(\infty)$ is invertible but is not a signature matrix, then, writing

$$
N(\infty)=K^{*} J K,
$$

where $J=J^{*}=J^{-1}$, we see that the function $K^{-*} N(z) K^{-1}$ satisfies the conditions of Theorem 2.22 .

\section{§3. Infinite-DimEnsiOnal $\mathcal{L}(N)$-SPACES}

In this section we study infinite-dimensional $\mathcal{L}(N)$-spaces. Now we cannot use the realization theory; instead, we employ the Riesz-Herglotz representation of a matrixvalued function $N$ analytic in the open upper half-plane and having a positive imaginary part there:

$$
N(z)=A+B z+\int_{\mathbb{R}}\left\{\frac{1}{t-z}-\frac{t}{t^{2}+1}\right\} d \mu(t)
$$

for a $\mathbb{C}^{n \times n}$-valued function $N$ analytic and with positive imaginary part in $\mathbb{C}_{+}$. In (3.1), the matrix $A$ is Hermitian, the matrix $B$ is positive, and $d \mu$ is a $\mathbb{C}^{n \times n}$-valued positive measure such that $\int_{\mathbb{R}} \frac{d \mu(t)}{t^{2}+1}<\infty$.

3.1. $\mathcal{L}(N)$-spaces. The reproducing-kernel Hilbert spaces associated with HerglotzNevanlinna functions (that is, the reproducing kernel of which is of the form $\frac{N(z)-N(w)^{*}}{z-\bar{w}}$ ) also play an important role in the present section. As before, we call them $\mathcal{L}(N)$-spaces. We start with reviewing their main properties.

First, we note that formula (3.1) implies that $N$ admits analytic extension to the open lower half-plane via

$$
N(z)=N(\bar{z})^{*} .
$$

Theorem 3.1. Let $N$ be a $\mathbb{C}^{n \times n}$-valued function analytic in the open upper-half plane $\mathbb{C}_{+}$. The following statements are equivalent:

1) $\operatorname{Im} N(z) \geq 0$ in $\mathbb{C}_{+}$;

2) the function $K_{N}(z, w)=\frac{N(z)-N(w)^{*}}{z-\bar{w}}$ is positive in $\mathbb{C}_{+}$.

Proof. Trivially, the second assertion implies the first. Starting from the Riesz-Herglotz representation formula for $N$, we obtain

$$
\frac{N(z)-N(w)^{*}}{z-\bar{w}}=B+\int_{\mathbb{R}} \frac{d \mu(t)}{(t-z)(t-\bar{w})},
$$

which shows that the kernel $K_{N}(z, w)$ is positive in $\mathbb{C}_{+}$. 
Extending $N$ to the open lower half-plane by (3.2), we see that the preceding result remains true in $\mathbb{C} \backslash \mathbb{R}$ if we replace the first condition by

$$
\frac{\operatorname{Im} N(z)}{\operatorname{Im} z} \geq 0
$$

Using (3.3), we arrive at the following statement.

Theorem 3.2. Let $N$ be a $\mathbb{C}^{n \times n}$-valued Herglotz-Nevanlinna function, and let (3.1) be its Riesz-Herglotz representation. Then the space $\mathcal{L}(N)$ consists of all functions of the form

$$
F(z)=B b+\int_{\mathbb{R}} \frac{d \mu(t) f(t)}{t-z}
$$

with $b \in \mathbb{C}^{n}$ and $f \in \mathbf{L}_{2}^{n}(d \mu)$, and the inner product in $\mathcal{L}(N)$ has the form

$$
\langle F, G\rangle_{\mathcal{L}(N)}=c^{*} B b+\int_{\mathbb{R}} g(t)^{*} d \mu(t) f(t),
$$

where $G(z)=B c+\int_{\mathbb{R}} \frac{d \mu(t) g(t)}{t-z}$.

For the proof of this result, we refer the reader to [19, 2]. It should be noted that an integral of the form $\int_{\mathbb{R}} \frac{d \mu(t) f(t)}{t-z}$ may converge for all nonreal $z$ for some $f$ not in $\mathbf{L}_{2}^{n}(d \mu)$. Then the function

$$
z \mapsto \int_{\mathbb{R}} \frac{d \mu(t) f(t)}{t-z}
$$

does not belong to $\mathcal{L}(N)$. For instance, take $d \mu(t)=\frac{d t}{t^{2}+1}$ and $f(t)=t$. Then $\int_{\mathbb{R}} \frac{t d t}{\left(t^{2}+1\right)(t-z)}$ exists for all nonreal $z$, but $\int_{\mathbb{R}} \frac{t^{2} d t}{t^{2}+1}=\infty$. Thus, the function $z \mapsto$ $\int_{\mathbb{R}} \frac{t d t}{\left(t^{2}+1\right)(t-z)}$ is not in $\mathcal{L}(N)$.

Proposition 3.3. Let $N$ be a $\mathbb{C}^{n \times n}$-valued Herglotz-Nevanlinna function and assume that $\operatorname{det} N \not \equiv 0$. Then the function $-N(z)^{-1}$ is well defined and is also a HerglotzNevanlinna function, and

$$
f \mapsto N f
$$

is a unitary map from $\mathcal{L}\left(-N^{-1}\right)$ onto $\mathcal{L}(N)$.

Proof. Let $w_{j} \in \mathbb{C} \backslash \mathbb{R}$, and let $e_{j} \in \mathbb{C}^{n}$ for $j=1, \ldots, M$. We have

$$
\begin{aligned}
N(z) & K_{-N^{-1}}\left(z, w_{j}\right) e_{j}=N(z) \frac{-N^{-1}(z)+N\left(w_{j}\right)^{-*}}{z-\overline{w_{j}}} e_{j} \\
& =\frac{-I_{n}+N(z) N\left(w_{j}\right)^{-*}}{z-\overline{w_{j}}} e_{j} \\
& =\frac{N(z)-N\left(w_{j}\right)^{*}}{z-\overline{w_{j}}} N\left(w_{j}\right)^{-*} e_{j} \\
& =K_{N}\left(z, w_{j}\right) N\left(w_{j}\right)^{-*} e_{j} .
\end{aligned}
$$

Thus, for $f(z)=\sum_{j=1}^{M} K_{-N^{-1}}\left(z, w_{j}\right) e_{j}$, the function

$$
N f(z)=\sum_{j=1}^{M} K_{N}\left(z, w_{j}\right) N\left(w_{j}\right)^{-*} e_{j}
$$


belongs to $\mathcal{L}(N)$, and

$$
\begin{aligned}
& \|N f\|_{\mathcal{L}(N)}^{2}=\sum_{\ell, j=1}^{M}\left\langle K_{N}\left(z, w_{j}\right) N\left(w_{j}\right)^{-*} e_{j}, K_{N}\left(z, w_{\ell}\right) N\left(w_{\ell}\right)^{-*} e_{\ell}\right\rangle_{\mathcal{L}(N)} \\
& =\sum_{\ell, j=1}^{M} e_{\ell}^{*} N\left(w_{\ell}\right)^{-1} K_{N}\left(w_{\ell}, w_{j}\right) N\left(w_{j}\right)^{-*} e_{j} \\
& =\sum_{\ell, j=1}^{M} e_{\ell}^{*} K_{-N^{-1}}\left(w_{\ell}, w_{j}\right) e_{j} \\
& =\|f\|_{\mathcal{L}\left(-N^{-1}\right)}^{2} .
\end{aligned}
$$

This completes the proof because such $f$ form a dense subset of $\mathcal{L}\left(-N^{-1}\right)$.

The case where the spaces $\mathcal{L}(N)$ and $\mathcal{L}\left(-N^{-1}\right)$ contain no nonzero constants will be of special interest in the sequel. This case can be characterized as follows.

Proposition 3.4. Let $N$ be a $\mathbb{C}^{n \times n}$-valued Herglotz-Nevanlinna function such that $\operatorname{det} N(z) \not \equiv 0$ in $\mathbb{C}_{+}$. The following statements are equivalent:

1) the spaces $\mathcal{L}(N)$ and $\mathcal{L}\left(-N^{-1}\right)$ contain no nonzero constant functions;

2) $\mathcal{L}(N)$ contains no nonzero constant function and no nonzero function of the form $z \mapsto N(z) c$ with $c \in \mathbb{C}^{n}$

3) in the representation (3.1), we have $B=0$, and if $\int_{\mathbb{R}} c^{*} d \mu(t) c<\infty$ for some $c \in \mathbb{C}^{n}$, then

$$
A c-\int_{\mathbb{R}} \frac{t d \mu(t) c}{t^{2}+1} \neq 0
$$

Proof. By the characterization (3.4), we have that $\mathcal{L}(N)$ contains no nonzero constants if and only if $B=0$ in (3.1). Now, assume that $\mathcal{L}(N)$ contains a function of the form $N(z) c$. Then there is a function $f \in \mathbf{L}_{2}^{n}(d \mu)$ such that

$$
N(z) c=\int_{\mathbb{R}} \frac{d \mu(t) f(t)}{t-z} .
$$

Applying the operator $R_{\alpha}$ to both sides of this equation (for some $\alpha \in \mathbb{C} \backslash \mathbb{R}$ ), we obtain

$$
\int_{\mathbb{R}} \frac{d \mu(t) c}{(t-z)(t-\alpha)}=\int_{\mathbb{R}} \frac{d \mu(t) f(t)}{(t-z)(t-\alpha)} .
$$

Consequently, $f(t)=c$ almost everywhere with respect to $\mu$, so that $f$ belongs to $\mathbf{L}_{2}(d \mu)$. Therefore, the integral $\int_{\mathbb{R}} d \mu(t) \frac{t}{t^{2}+1}$ exists by the Cauchy-Schwarz inequality, and 3.6 becomes

$$
A c+\int_{\mathbb{R}} d \mu(t) c \frac{1}{t-z}-\int_{\mathbb{B}} d \mu(t) c \frac{t}{t^{2}+1}=\int_{\mathbb{R}} \frac{d \mu(t) f(t)}{t-z} .
$$

It follows that $A c=\int_{\mathbb{R}} d \mu(t) c \frac{t}{t^{2}+1}$.

Similar arguments were used in [1].

The class of Herglotz-Nevanlinna functions appearing in Proposition 3.4 with equality rather than inequality in (3.5) was introduced (via the third characterization) by Bely and Tsekanovskiu in [15, p. 65]. They denoted that class by $N(R)$. This is the largest class of Herglotz-Nevanlinna functions that admit a realization in terms of a BrodskiuLivshitz colligation. The main operator of this colligation is defined between rigged Hilbert spaces; see [1] and [16, p. 98]. 
Thus, our class and that of Belyı̆ and Tsekanovskiı are complementary in a sense. Their characterization of $N(R)$ is as follows.

Theorem 3.5. A matrix-valued Herglotz-Nevanlinna function $N$ is in the class $N(R)$ if and only if it can be written as

$$
N(z)=i\left(W_{\theta}(z)+I\right)^{-1}\left(W_{\theta}(z)-I\right) J
$$

where

$$
W_{\theta}(z)=I-2 i K^{*}(A-z I)^{-1} K J .
$$

In this expression, $A$ is a correct (*)-extension of an operator $T$ in $\Omega_{A}$ and

$$
A-A^{*}=2 i K J K^{*} \text {. }
$$

We refer the reader to [1] and [16, p. 98] for the definitions of the classes $\Omega_{A}$ and of a correct $(*)$-extension of an operator $T$.

Another criterion for $\mathcal{L}(N)$ to contain no nonzero constant functions is as follows.

Proposition 3.6. Let $N$ be a Herglotz-Nevanlinna function. Then $\mathcal{L}(N)$ contains no nonzero constant functions if and only if

$$
\lim _{y \rightarrow+\infty} \frac{N(i y)}{i y}=0 .
$$

Proof. Let (3.1) be the Riesz-Herglotz representation of $N$. Then

$$
\frac{N(i y)}{i y}=\frac{A}{i y}+B+\int_{\mathbb{R}} \frac{d \mu(t)}{t^{2}+1}\left\{\frac{i y t+1}{(i y)(t-i y)}\right\} \text {. }
$$

Since

we have

$$
\left|\frac{i y t+1}{(i y)(t-i y)}\right|^{2}=\frac{t^{2} y^{2}+1}{y^{2}\left(t^{2}+y^{2}\right)} \leq 1 \quad \text { for } y \geq 1
$$

$$
\lim _{y \rightarrow+\infty} \frac{N(i y)}{i y}=B
$$

by the dominated convergence theorem, whence the result.

We note that condition (3.7) is necessary and sufficient for a function to be the $Q$ function of a Hermitian operator. See [36, Hauptsatz on p. 203] and \$7]

The map $f \mapsto N f$ induces a related map in the Lebesgue spaces.

Theorem 3.7. Let $N$ be a $\mathbb{C}^{n \times n}$-valued Herglotz-Nevanlinna function. Assume that $\operatorname{det} N(z) \not \equiv 0$ and that the spaces $\mathcal{L}(N)$ and $\mathcal{L}\left(-N^{-1}\right)$ do not contain nonzero constant functions. Let

$$
\begin{aligned}
N(z) & =A_{+}+\int_{\mathbb{R}}\left\{\frac{1}{t-z}-\frac{t}{t^{2}+1}\right\} d \mu_{+}(t), \\
-N(z)^{-1} & =A_{-}+\int_{\mathbb{R}}\left\{\frac{1}{t-z}-\frac{t}{t^{2}+1}\right\} d \mu_{-}(t)
\end{aligned}
$$

be the Riesz-Herglotz representations of $N$ and $-N^{-1}$, respectively. Then for any $f_{-} \in$ $\mathbf{L}_{2}\left(d \mu_{-}\right)$there exists a unique $f_{+} \in \mathbf{L}_{2}\left(d \mu_{+}\right)$such that

$$
N(z) \int_{\mathbb{R}} \frac{d \mu_{-}(t) f_{-}(z)}{t-z}=\int_{\mathbb{R}} \frac{d \mu_{+}(t) f_{+}(z)}{t-z} .
$$

See [18] for the proof. The map $f_{-} \mapsto f_{+}$is called the Hilbert transformation. We refer the reader to our earlier paper [8] and to Subsections 3.3] and 6.1] for the case where $N$ is in the Wiener algebra. 
3.2. $\mathcal{L}(N)$-spaces and operator models. First, we recall the following result (see [18]), the proof of which is provided for completeness.

Theorem 3.8. Let $\mathcal{H}$ be a reproducing-kernel Hilbert space of functions analytic in $\mathbb{C} \backslash \mathbb{R}$. Then its reproducing kernel is of the form $K_{N}(z, w)$ if and only if the following conditions are fulfilled:

1) $R_{\alpha} \mathcal{H} \subset \mathcal{H}$ for all $\alpha \in \mathbb{C} \backslash \mathbb{R}$

2) for all $\alpha, \beta \in \mathbb{C} \backslash \mathbb{R}$ and all $f, g \in \mathcal{H}$ we have

$$
\left\langle R_{\alpha} f, g\right\rangle-\left\langle f, R_{\beta} g\right\rangle-(\alpha-\bar{\beta})\left\langle R_{\alpha} f, R_{\beta} g\right\rangle=0 .
$$

Proof. To simplify notation, we consider the case of scalar-valued functions. One direction follows from formula (2.7), which allows us to compute various inner products in (3.8) at linear combinations of kernels. More precisely, let $f(z)=K_{N}(z, w)$, and let $g(z)=K_{N}(z, v)$. Using (2.7), we obtain the relations

$$
\begin{aligned}
\left\langle R_{\alpha} f, g\right\rangle & =\frac{1}{\alpha-\bar{w}}\left(K_{N}(\bar{\alpha}, v)-K_{N}(w, v)\right), \\
\left\langle f, R_{\beta} g\right\rangle & =\frac{1}{\bar{\beta}-v}\left(K_{N}(\bar{\beta}, w)-K_{N}(v, w)\right), \\
\left\langle R_{\alpha} f, R_{\beta} g\right\rangle & =\frac{1}{(\alpha-\bar{w})(\bar{\beta}-v)}\left(K_{N}(\bar{\beta}, \bar{\alpha})-K_{N}(\bar{\beta}, w)-K_{N}(v, \bar{\alpha})+K_{N}(v, w)\right) .
\end{aligned}
$$

Using these expressions, we see that the proof of (3.8) amounts to the proof of the identity

$$
(\alpha-v) K_{N}(v, \bar{\alpha})+(\bar{w}-\bar{\beta}) K_{N}(\bar{\beta}, w)-(\alpha-\bar{\beta}) K(\bar{\beta}, \bar{\alpha})+(\bar{w}-v) K_{N}(v, w)=0 .
$$

In its turn, this is a direct consequence of the form of $K_{N}(z, w)$.

The converse is proved as follows (we still consider the scalar case for simplicity of notation). We choose $\beta=\bar{\alpha}, f(z)=K(z, w)$ and $g(z)=K(z, v)$ in (3.8) to obtain

$$
\frac{K(v, w)-K(\alpha, w)}{v-\alpha}=\left(\frac{K(w, v)-K(\bar{\alpha}, v)}{w-\bar{\alpha}}\right)^{*} .
$$

Thus, we get

$$
(v-\bar{w}) K(v, w)=(v-\alpha) K(v, \bar{\alpha})-(\bar{w}-\alpha) K(\alpha, w) .
$$

Setting $w=\alpha$ leads to

$$
(v-\bar{\alpha}) K(v, \alpha)=(v-\alpha) K(v, \bar{\alpha})-(\bar{\alpha}-\alpha) K(\alpha, \alpha) .
$$

Set

$$
N(z)=(v-\alpha) K(v, \bar{\alpha})+\frac{\alpha-\bar{\alpha}}{2} K(\alpha, \alpha) .
$$

Using (3.9), we obtain

$$
(v-\bar{w}) K(v, w)=N(v)-N(w)^{*},
$$

and the result follows.

If the operators $R_{\alpha}$ have no kernel, then there is a (possibly unbounded) operator $G_{+}$ such that $R_{\alpha}=\left(G_{+}-\alpha I\right)^{-1}$. Identity (3.8) forces $G_{+}$to be selfadjoint.

Theorem 3.9. Let $G_{+}$be a densely defined selfadjoint operator in a Hilbert space $\mathcal{H}$. We assume that there is an operator $C: \mathcal{H} \rightarrow \mathbb{C}^{n}$ such that (4.1) is true,

$$
\bigcap_{z \in \mathbb{C} \backslash \mathbb{R}} \operatorname{ker} C\left(G_{+}-z I\right)^{-1}=\{0\},
$$

and we put $N(z)=D+C\left(G_{+}-z I\right)^{-1} C^{*}$. Let

$$
N(z)=D+\int_{\mathbb{R}} d \mu(t)\left\{\frac{1}{t-z}-\frac{t}{t^{2}+1}\right\}
$$


be the Riesz-Herglotz representation of $N$. The map that takes $h \in \mathcal{H}$ to the function $f \in \mathbf{L}_{2}^{n}(d \mu)$ such that

$$
C\left(G_{+}-z I\right)^{-1} h=\int_{\mathbb{R}} \frac{d \mu(t) f(t)}{t-z}
$$

is unitary. Finally, $G_{+}$is unitarily equivalent under the map (3.10) to the operator of multiplication by the independent variable in $\mathbf{L}_{2}(d \mu)$

Proof. This follows from the relation

$$
\int_{\mathbb{R}} \frac{d \mu(t) t f(t)}{t-z}=z \int_{\mathbb{R}} \frac{d \mu(t) f(t)}{t-z}+\int_{\mathbb{R}} d \mu(t) f(t) .
$$

We note that

$$
-\lim _{z \rightarrow \infty} z F(z)=\int_{\mathbb{R}} d \mu(t) f(t) .
$$

Finally, it is of interest to consider Theorem 3.8 in the finite-dimensional case. Since the space $\mathcal{H}$ is $R_{\alpha}$-invariant, it is formed by rational functions (see Subsection 2.1). Provided these functions are analytic at infinity, a basis of $\mathcal{H}$ consists of the columns of a matrix-valued function of the form $C\left(z I_{m}-A\right)^{-1}$, where $m=\operatorname{dim} \mathcal{H}$. Let $P$ be the Gram matrix of $\mathcal{H}$ with respect to this basis. Then the structural equation (3.8) becomes $A^{*} P=P A$. We recognize equation (2.8) with $H=P^{-1}$, and it is easy to check that $P^{-1}$ is indeed the matrix associated with a minimal realization of $N$.

3.3. The case where $N$ belongs to the Wiener algebra. The Wiener algebra $\mathcal{W}^{n \times n}$ consists of the functions of the form

$$
f(z)=D+\int_{-\infty}^{\infty} e^{i z t} u(t) d t
$$

where $D \in \mathbb{C}^{n \times n}$ and $u \in \mathbf{L}_{1}^{n \times n}(\mathbb{R})$. The subalgebra $\mathcal{W}_{+}^{n \times n}$ (respectively, $\mathcal{W}_{-}^{n \times n}$ ) consists of all functions of the form (3.11) for which the support of $u$ is in $\mathbb{R}_{+}$(respectively, in $\mathbb{R}_{-}$).

In the case where $N$ belongs to $\mathcal{W}_{+}^{n \times n}$, we proved in 8$]$ that $f_{-}$is given by the formula

$$
f_{-}(z)=i p N^{*} p f-i q N q f,
$$

where $p=I-q$ denotes the orthogonal projection from the Lebesgue space $\mathbf{L}_{2}^{n}(\mathbb{R})$ onto the Hardy space in the open upper half-plane.

\section{$\S 4$. The CASE OF BOUNDED OPERATORS}

In this section we turn to the case of bounded selfadjoint operators in possibly infinitedimensional spaces. The analysis is very close to the case of matrices. The main difference is that in the study of reproducing-kernel Hilbert spaces with reproducing kernel of the form $\frac{N(z)-N(w)^{*}}{z-\bar{w}}$ we cannot use the realization theory; instead, we apply the RieszHerglotz representation formula (3.1).

4.1. Models for a bounded selfadjoint operator. First, we explain how spaces of the form $\mathcal{L}(N)$ give models for selfadjoint operators. For more details, see [18]. In this section we focus on the case where $G_{+}$is bounded. The case of possibly unbounded operators is considered in $\$ 5$

Theorem 4.1. Let $G_{+}$be a bounded selfadjoint operator on a Hilbert space $\mathcal{H}$. Assume that there is an operator $C: \mathcal{H} \rightarrow \mathbb{C}^{n}$ such that

$$
\bigcap_{z \in \mathbb{C} \backslash \mathbb{R}} \operatorname{ker} C\left(G_{+}-z I\right)^{-1}=\{0\} .
$$


For $h \in \mathcal{H}$, we define

$$
\widehat{h}(z)=C\left(G_{+}-z I\right)^{-1} h, \quad z \in \mathbb{C} \backslash \mathbb{R} .
$$

Then $\widehat{h}(z) \equiv 0 \Longleftrightarrow h=0$, and the set of functions of the form (4.2) endowed with the norm

$$
\|\widehat{h}\| \stackrel{\text { def }}{=}\|h\|_{\mathcal{H}}
$$

is a reproducing-kernel Hilbert space of functions analytic in $\mathbb{C} \backslash \mathbb{R}$. The reproducing kernel has the form $\frac{N(z)-N(w)^{*}}{z-\bar{w}}$, where

$$
N(z)=D+C\left(G_{+}-z I\right)^{-1} C^{*},
$$

$D$ being any selfadjoint matrix in $\mathbb{C}^{n \times n}$. Furthermore, the map $h \mapsto \widehat{h}$ takes the operator $\left(G_{+}-\alpha I\right)^{-1}$ with $\alpha \in \mathbb{C} \backslash \mathbb{R}$ to the operator $R_{\alpha}$ :

$$
\left(\widehat{G_{+}-\alpha}\right)^{-1} h(z)=\frac{\widehat{h}(z)-\widehat{h}(\alpha)}{z-\alpha} .
$$

Proof. Let $\widehat{\mathcal{H}}$ denote the set of functions of the form (4.2); relation (4.1) shows that formula (4.3) determines a norm. For every $c \in \mathbb{C}^{n}$, the function

$$
z \mapsto K(z, w) c=C\left(G_{+}-z I\right)^{-1}\left(G_{+}-w I\right)^{-*} C^{*} c
$$

belongs to $\widehat{\mathcal{H}}$. Furthermore, for every $h \in \mathcal{H}$ we have

$$
\begin{aligned}
& \langle\widehat{h}, K(z, w) c\rangle_{\widehat{\mathcal{H}}}=\left\langle h,\left(G_{+}-w I\right)^{-*} C^{*} c\right\rangle_{\mathcal{H}} \\
& \quad=\left\langle C\left(G_{+}-w I\right)^{-1} h, c\right\rangle_{\mathbb{C}^{n}} \\
& \quad=c^{*} \widehat{h}(w),
\end{aligned}
$$

so that $\widehat{\mathcal{H}}$ is the reproducing-kernel Hilbert space with the reproducing kernel

$$
C\left(G_{+}-z I\right)^{-1}\left(G_{+}-w I\right)^{-*} C^{*} .
$$

Now, let $N$ be defined by (4.4). Then $N$ is analytic in $\mathbb{C} \backslash \mathbb{R}$, and we have

$$
\begin{aligned}
N(z) & -N(w)^{*}=C\left(\left(G_{+}-z I\right)^{-1}-\left(G_{+}-\bar{w} I\right)^{-1}\right) C^{*} \\
& =(z-\bar{w}) C\left(G_{+}-z I\right)^{-1}\left(G_{+}-w I\right)^{-*} C^{*}
\end{aligned}
$$

for all $z, w \in \mathbb{C} \backslash \mathbb{R}$, whence the result.

The space $\mathcal{L}(N)$ in the preceding theorem contains no nonzero constants. Indeed, by (4.5), a constant function corresponds to an element in the kernel of $\left(G_{+}-\alpha I\right)^{-1}$ for any nonreal point $\alpha$. If $N$ is a $\mathbb{C}^{n \times n}$-valued function analytic and with a positive real part in the open upper half-plane $\mathbb{C}_{+}$(i.e., $N$ is a Herglotz-Nevanlinna function) and $\operatorname{det} N(z) \neq$ 0 , then $-N^{-1}$ is also a Herglotz-Nevanlinna function. If the space $\mathcal{L}\left(-N^{-1}\right)$ contains no nonzero constants, the operators $R_{\alpha}$ are the resolvents of a selfadjoint operator. So, in this case there are two selfadjoint operators associated with $N$ in a natural way. This is the idea behind the result of de Branges and Rovnyak that gives a model for a pair of selfadjoint operators in terms of a single Herglotz-Nevanlinna function. 
4.2. The model of de Branges and Rovnyak. We write

$$
G_{+}-G_{-}=-C J C^{*}
$$

where $J$ is a signature matrix of size $n \times n$ (that is, $J$ is both selfadjoint and unitary), and define $N$ by (2.28). Then the computations of $\S 2$ go through without changes up to Proposition 2.20, Using the spectral theorem for bounded selfadjoint operators, we set

$$
N(z)=J+\int_{\mathbb{R}} \frac{d \mu_{+}(t)}{t-z} .
$$

Now, we present a counterpart of Theorem 2.17 We present it in terms of Lebesgue spaces; the passage to the $\mathcal{L}(N)$-spaces is left to the reader.

We set

$$
V_{+} f(z)=\int_{\mathbb{R}} \frac{d \mu_{+}(t) f(t)}{t-z}
$$

and similarly for $-N^{-1}$. We begin with a preliminary lemma.

Lemma 4.2. Let $d \mu$ be a $\mathbb{C}^{n \times n}$-valued positive measure on $\mathbb{R}$. Then the operator of multiplication by $t$ is bounded in $\mathbf{L}_{2}^{n}(d \mu)$ if and only if $d \mu$ has bounded support.

Proof. Assume that the support of $d \mu$ is unbounded, say toward $+\infty$. Then for every $n>0$ there exists a function $f \in \mathbf{L}_{2}^{n}(d \mu)$ with support on $[n,+\infty)$. Thus,

$$
\int_{\mathbb{R}} t^{2} f(t)^{*} d \mu(t) f(t) \geq n^{2} \int_{\mathbb{R}} f(t)^{*} d \mu(t) f(t),
$$

and we see that the norm of the operator of multiplication by $t$ is at least $n$ for all $n$. Therefore, this operator is unbounded. The converse is clear because

$$
\int_{\mathbb{R}} t^{2} f(t)^{*} d \mu(t) f(t) d t \leq M \int_{\mathbb{R}} f(t)^{*} d \mu(t) f(t) d t,
$$

where $M$ is such that the support of $d \mu$ is inside $[-M, M]$.

This lemma shows that all the operators in the next theorem are bounded.

Theorem 4.3. For the map $V_{+}$, we have

$$
\begin{aligned}
& V_{+} G_{+} V_{+}^{*} f(t)=t f(t), \\
& V_{+} G_{-} V_{+}^{*} f(t)=t f(t)+J \int_{\mathbb{R}} d \mu_{+}(t) f(t), \\
& V_{+}\left(G_{+}-\alpha I_{m}\right)^{-1} V_{+}^{*} f(t)=\frac{f(t)}{t-\alpha}, \\
& V_{+}\left(G_{-}-\alpha I_{m}\right)^{-1} V_{+}^{*} f(t)=\frac{f(t)}{t-\alpha}-\left(J+\int_{\mathbb{R}} \frac{d \mu_{+}(s)}{s-\alpha}\right)^{-1} \frac{\int_{\mathbb{R}} \frac{d \mu_{+}(s) f(s)}{s-\alpha}}{t-\alpha},
\end{aligned}
$$

where $f \in \mathbf{L}_{2}\left(d \mu_{+}\right)$. In particular,

$$
\begin{aligned}
& V_{+}\left\{\left(G_{+}-\alpha I_{m}\right)^{-1}-\left(G_{-}-\alpha I_{m}\right)^{-1}\right\} V_{+}^{*} F \\
& \quad=\frac{1}{t-\alpha}\left(J+\int_{\mathbb{R}} \frac{d \mu_{+}(s)}{s-\alpha}\right)^{-1} \int_{\mathbb{R}} \frac{d \mu_{+}(s) f(s)}{s-\alpha} .
\end{aligned}
$$


Similarly, for the map $V_{-}$we have

$$
\begin{aligned}
& V_{-} G_{-} V_{-}^{*} f(t)=t f(t), \\
& V_{-} G_{+} V_{-}^{*} F(z)=t f(t)-J \int_{\mathbb{R}} d \mu_{-}(t) f(t), \\
& V_{-}\left(G_{-}-\alpha I_{m}\right)^{-1} V_{-}^{*} f(t)=\frac{f(t)}{t-\alpha}, \\
& V_{-}\left(G_{+}-\alpha I_{m}\right)^{-1} V_{-}^{*} F \\
& \quad=\frac{f(t)}{t-\alpha}-\left(J+\int_{\mathbb{R}} \frac{d \mu_{+}(t)}{t-\alpha}\right) \frac{\int_{\mathbb{R}} d \mu_{-}(t) f(t)}{t-\alpha}, \quad f \in \mathbf{L}_{2}\left(d \mu_{-}\right) .
\end{aligned}
$$

In particular,

$$
\begin{aligned}
& V_{-}\left\{\left(G_{+}-\alpha I_{m}\right)^{-1}-\left(G_{-}-\alpha I_{m}\right)^{-1}\right\} V_{-}^{*} F \\
& =-\frac{1}{t-\alpha}\left(J+\int_{\mathbb{R}} \frac{d \mu_{+}(t)}{t-\alpha}\right) \int_{\mathbb{R}} d \mu_{-}(t) f(t),
\end{aligned}
$$

where $f \in \mathbf{L}_{2}\left(d \mu_{-}\right)$.

This theorem is a counterpart of Theorem 2.17 the proof is similar and is omitted.

4.3. The trace formula for pairs of bounded selfadjoint operators. Only the second proof of the determinant formula (2.38) remains valid.

Proposition 2.21 cannot be proved in the same way as in $\S 2$. We discuss it, distinguishing two cases: the scalar case and the matrix-valued case. Now, in the scalar case, relationship with Krein's formula is established by using the following result of N. Aronszajn and W. Donoghue.

Proposition 4.4. A function $N$ is a Herglotz-Nevanlinna function if and only if it can be written as

$$
N(z)=\exp \left\{\int_{\mathbb{R}}\left(\frac{1}{t-z}-\frac{t}{t^{2}+1}\right) f(t) d t\right\}
$$

where $C>0$ and $f$ is a measurable function such that $0 \leq f(t) \leq 1$ almost everywhere on $\mathbb{R}$ and $\int_{\mathbb{R}} \frac{f(t) d t}{t^{2}+1}<\infty$.

See [13, 12, [24, p. 27], and [37, Theorem A.3, p. 391].

As a consequence, we have

$$
\frac{N^{\prime}(z)}{N(z)}=\int_{\mathbb{R}} \frac{f(t) d t}{(t-z)^{2}}
$$

The result of Aronszajn and Donoghue was extended to the matrix-valued (and even operator-valued) case by Carey [22] and by Gesztesy, Makarov, and Naboko [27].

Theorem 4.5. Let $N$ be a $\mathbb{C}^{n \times n}$-valued function analytic in the open upper half-plane and such that $\operatorname{Im} N(z)>0$ for $\operatorname{Im} z>0$ and $N(\infty)=I_{n}$. Then there exists a measurable $\mathbb{C}^{n \times n}$-valued function $B(t)$ such that $0 \leq B(t) \leq I_{n}$ and

$$
N(z)=\exp \left\{\int_{\mathbb{R}} \frac{B(t) d t}{t-z}\right\} .
$$

We briefly discuss this result.

Lemma 4.6. Let $F(z)=\exp A(z)$, where $A$ is a $\mathbb{C}^{n \times n}$-valued function analytic in some domain $\Omega$. Then

$$
\operatorname{Tr}\left(F^{-1}(z) F^{\prime}(z)\right)=\operatorname{Tr} A^{\prime}(z) .
$$


Proof. We have

$$
\begin{aligned}
F^{\prime}(z)= & A^{\prime}(z)+\frac{1}{2 !}\left(A(z) A^{\prime}(z)+A^{\prime}(z) A(z)\right) \\
& +\frac{1}{3 !}\left(A^{2}(z) A^{\prime}(z)+A(z) A^{\prime}(z) A(z)+A^{\prime}(z) A^{2}(z)\right)+\cdots
\end{aligned}
$$

and

Therefore,

$$
F^{-1}(z)=I_{n}-A(z)+\frac{1}{2 !} A^{2}(z)-\cdots .
$$

$$
F^{-1}(z) F^{\prime}(z)=A^{\prime}(z)+\left(-A(z) A^{\prime}(z)+\frac{1}{2 !}\left(A(z) A^{\prime}(z)+A^{\prime}(z) A(z)\right)\right)+\cdots,
$$

and (4.12) follows by taking the trace of both sides.

This lemma shows that (4.11) implies the formula

$$
\operatorname{Tr} N^{-1}(w) N^{\prime}(w)=\int_{\mathbb{R}} \frac{\operatorname{Tr} B(t) d t}{(t-w)^{2}} .
$$

Lemma 4.7. Let $N$ be a $\mathbb{C}^{n \times n}$-valued function analytic in the open upper half-plane $\mathbb{C}_{+}$ and such that $\operatorname{Im} N(z)>0$ for $\operatorname{Im} z>0$. Then there exists $a \mathbb{C}^{n \times n}$-valued function $L(z)$ analytic in the open upper half-plane and such that

$$
N(z)=\exp L(z) .
$$

Proof. The function $S(z)=\left(I_{n}+i N(z)\right)\left(I_{n}-i N(z)\right)^{-1}$ is analytic and strictly contractive in $\mathbb{C}_{+}$. Thus,

$$
I_{n}-S(z)=\exp L_{1}(z) \quad \text { with } L_{1}(z)=-\left(\sum_{j=1}^{\infty} \frac{S(z)^{j}}{j}\right)
$$

Similarly,

$$
I_{n}+S(z)=\exp L_{2}(z) \quad \text { with } L_{2}(z)=-\left(\sum_{j=1}^{\infty} \frac{(-1)^{j} S(z)^{j}}{j}\right) .
$$

It follows that

$$
\begin{aligned}
N(z) & =i\left(I_{n}-S(z)\right)\left(I_{n}+S(z)\right)^{-1}=i \exp L_{2}(z) \exp -L_{1}(z) \\
& =\exp \left\{\frac{\pi}{2} I_{n}+L_{1}(z)-L_{2}(z)\right\}
\end{aligned}
$$

since $L_{1}$ and $L_{2}$ commute. This completes the proof because $L_{1}$ and $L_{2}$ are analytic in $\mathbb{C}_{+}$and

$$
L(z)=\frac{\pi}{2} I_{n}-\sum_{j=0}^{\infty} \frac{S(z)^{2 j+1}}{2 j+1} .
$$

To indicate links with the results by Carey and by Gesztesy, Makarov, and Naboko mentioned above, it remains to show that $L(z)$ can be written in the form $L(z)=$ $\int_{\mathbb{R}} \frac{C(t) d t}{t-z}$. In the next lemma we prove a little less.

Lemma 4.8. Let $L$ be a $\mathbb{C}^{n \times n}$-valued function analytic in $\mathbb{C}_{+}$and such that $L(i)=0$ and the function $\frac{L(z)}{z+i}$ has its entries in the Hardy space of the open upper half-plane. Then

$$
L(z)=\int_{\mathbb{R}} L(t)\left\{\frac{1}{t-z}-\frac{t}{t^{2}+1}\right\} d t .
$$


Proof. We have

$$
\begin{aligned}
& \int_{\mathbb{R}} L(t)\left\{\frac{1}{t-z}-\frac{t}{t^{2}+1}\right\} d t=\int_{\mathbb{R}} \frac{L(t)}{t+i}\left\{\frac{t+i}{t-z}-\frac{t(t+i)}{t^{2}+1}\right\} d t \\
&=\int_{\mathbb{R}} \frac{L(t)}{t+i}\left\{\frac{z+i}{t-z}-\frac{i}{t-i}\right\} d t .
\end{aligned}
$$

Using Cauchy's formula for functions in the Hardy space, we obtain

$$
\int_{\mathbb{R}} L(t)\left\{\frac{1}{t-z}-\frac{t}{t^{2}+1}\right\} d t=\frac{L(z)}{z+i}(z+i)-i \frac{L(i)}{2 i}=L(z) .
$$

In particular, this lemma will be applicable if $\operatorname{Im} N(z) \geq x I_{n}$ for $z \in \mathbb{C}_{+}$, where $x>0$ is independent of $z$.

4.4. Inverse problems. In this subsection we give two characterizations of the characteristic functions of bounded selfadjoint operators.

Theorem 4.9. A Herglotz-Nevanlinna function $N$ is the characteristic function of a pair of bounded selfadjoint operators if and only if there is $M \geq 0$ such that after extension to the lower half-plane by $N(z)=N(\bar{z})^{*}$, the function $N$ becomes analytic in $\mathbb{C} \backslash[-M, M]$, and the following conditions are fulfilled:

$$
\begin{array}{r}
\lim _{y \rightarrow+\infty} \frac{N(i y)}{y}=0, \\
\lim _{y \rightarrow+\infty} \frac{N^{-1}(i y)}{y}=0,
\end{array}
$$

and $N(\infty)=J$. Under these conditions, a pair $\left(G_{+}, G_{-}\right)$of operators for which $N$ is the characteristic function is obtained as follows:

$$
\begin{array}{ll}
G_{+} F(z)=z F(z)+c_{F}, & F \in \mathcal{L}(N), \\
G_{-} F(z)=z F(z)+c_{F}, & F \in \mathcal{L}\left(-N^{-1}\right) .
\end{array}
$$

Proof. The Herglotz-Nevanlinna function $N$ is the characteristic function of a pair of bounded selfadjoint operators if and only if the spaces $\mathcal{L}(N)$ and $\mathcal{L}\left(-N^{-1}\right)$ both contain no nonzero constant functions. By Proposition 3.6] this is equivalent to (4.13) and (4.14).

The first requirement in the theorem is equivalent to the statement that the measure $d \mu_{+}$in the Riesz-Herglotz representation of $N$ has finite support, and thus the operator $G_{+}$is bounded in $\mathcal{L}(N)$. By the Cauchy-Schwarz inequality, the quantity

$$
c_{F}=\int_{\mathbb{R}} d \mu_{+}(t) f(t)
$$

is well defined for all $f \in \mathbf{L}_{2}^{n}\left(d \mu_{+}\right)$, so that

$$
G_{+} F(z)=\int_{\mathbb{R}} \frac{d \mu_{+}(t) f(t) t}{t-z}=\int_{\mathbb{R}} \frac{d \mu_{+}(t) f(t)(t-z+z)}{t-z}=z F(z)+c_{F} .
$$

From (4.14) it follows that

$$
\lim _{y \rightarrow \infty} y \operatorname{Im} N(i y)^{-1}<\infty
$$

Hence, $G_{-}$is also bounded.

We present an alternative characterization. 
Theorem 4.10. A Herglotz-Nevanlinna function $N$ is the characteristic function of a pair of bounded selfadjoint operators if and only if it can be written as

$$
N(z)=J+\int_{\mathbb{R}} \frac{d \mu_{+}(t)}{t-z},
$$

where $d \mu_{+}$is a positive measure with finite support and satisfying the condition

$$
J \neq \int_{\mathbb{R}} \frac{t d \mu_{+}(t)}{t^{2}+1} .
$$

A pair $\left(G_{+}, G_{-}\right)$can then be chosen as follows:

$$
G_{+} f(t)=t f(t) \quad \text { and } \quad G_{-} f(t)=t f(t)+J \int_{\mathbb{R}} d \mu_{+}(t) f(t),
$$

where $f \in \mathbf{L}_{2}\left(d \mu_{+}\right)$.

Equation (4.15) is the specialization of (3.5) to a finite measure.

\section{$\S 5$. The Case of Possibly unbounded operators}

In this section we consider operators that may be unbounded. Of particular interest is the case where $G_{+}$and $G_{-}$are common selfadjoint extensions of a given Hermitian operator. This latter case is considered in $\S 7$

\subsection{An operator model for pairs.}

Lemma 5.1. Let $G_{+}$and $G_{-}$be two selfadjoint operators. Then the space

$$
\mathcal{E}=\bigcap_{w \in \mathbb{C} \backslash \mathbb{R}} \operatorname{ker}\left\{\left(G_{+}-w I\right)^{-1}-\left(G_{-}-w I\right)^{-1}\right\}
$$

is the largest subspace with the following two properties: for all $w_{0} \in \mathbb{C} \backslash \mathbb{R}$, we have

$$
\left(G_{+}-w_{0} I\right)^{-1} \mathcal{E} \subset \mathcal{E} \quad \text { and } \quad\left(G_{-}-w_{0} I\right)^{-1} \mathcal{E} \subset \mathcal{E},
$$

and for all $f \in \mathcal{E}$ and some $w_{0} \in \mathbb{C} \backslash \mathbb{R}$ we have

$$
\left(G_{+}-w_{0} I\right)^{-1} f=\left(G_{-}-w_{0} I\right)^{-1} f .
$$

Proof. Let $\mathcal{M}$ be a closed subspace of $\mathcal{H}$ for which (5.1) and (5.2) are fulfilled. Then the resolvent identity implies that (5.2) is true for all $w \in \mathbb{C} \backslash \mathbb{R}$; indeed, (5.2) implies that

$$
\left(G_{+}-w I\right)^{-1}\left(G_{+}-w_{0} I\right)^{-1} f=\left(G_{+}-w I\right)^{-1}\left(G_{-}-w_{0}\right)^{-1} f,
$$

and since $\left(G_{-}-w_{0}\right)^{-1} f \in \mathcal{M}$, by (5.1) we have

$$
\left(G_{+}-w I\right)^{-1}\left(G_{+}-w_{0} I\right)^{-1} f=\left(G_{-}-w I\right)^{-1}\left(G_{-}-w_{0}\right)^{-1} f .
$$

Using the resolvent identity, we obtain

$$
\left(G_{+}-w I\right)^{-1} f-\left(G_{+}-w_{0} I\right)^{-1} f=\left(G_{-}-w I\right)^{-1} f-\left(G_{-}-w_{0} I\right)^{-1} f
$$

whence

$$
\left(G_{+}-w I\right)^{-1} f=\left(G_{-}-w I\right)^{-1} f .
$$

Thus, $f \in \mathcal{E}$, that is, we have $\mathcal{M} \subset \mathcal{E}$. The space $\mathcal{E}$ is easily seen to satisfy conditions (5.1) and (5.2), and this ends the proof. 
We note that $\mathcal{E}$ may be reduced to $\{0\}$, while $G_{+}$and $G_{-}$coincide on a dense set. This will happen, for instance, if $G_{+}$and $G_{-}$are canonical extensions of a common simple closed Hermitian operator.

We begin with the proof of Theorem [1.1. To ease the reading, we repeat the theorem (with the notation $G_{ \pm}$rather than $H_{ \pm}$).

Theorem 1.1. Let $\left(G_{+}, G_{-}\right)$be a pair of selfadjoint operators defined on a separable Hilbert space and having the following two properties:

1) there is $n \in \mathbb{N}$ such that

$$
\operatorname{dim} \operatorname{ran}\left(\left(G_{+}-w I\right)^{-1}-\left(G_{-}-w I\right)^{-1}\right)=n
$$

2)

$$
\bigcap_{w \in \mathbb{C} \backslash \mathbb{R}} \operatorname{ker}\left(\left(G_{+}-w I\right)^{-1}-\left(G_{-}-w I\right)^{-1}\right)=\{0\} .
$$

Then there exists a $\mathbb{C}^{n \times n}$-valued Herglotz-Nevanlinna function with $\operatorname{det} N \neq 0$ and with the following properties:

1) there are unitary transformations

$$
U_{+}: \mathcal{H} \rightarrow \mathcal{L}(N) \quad \text { and } \quad U_{-}: \mathcal{H} \rightarrow \mathcal{L}\left(-N^{-1}\right)
$$

such that

$$
\begin{aligned}
& U_{+}\left(G_{+}-w I\right)^{-1} f=R_{w} U_{+} f, \\
& U_{-}\left(G_{-}-w I\right)^{-1} f=R_{w} U_{-} f,
\end{aligned}
$$

for $w \in \mathbb{C} \backslash \mathbb{R}$, where $R_{w}$ is the resolvent-like operator defined by $R_{w} f(z)=$ $\frac{f(z)-f(w)}{z-w}$

2) for every $f \in \mathcal{H}$ we have

$$
\mathcal{M}_{-N^{-1}} U_{+} f=U_{-} f
$$

where $\mathcal{M}_{-N^{-1}}$ denotes the operator of multiplication by $-N^{-1}$ from the left.

As has already been mentioned, this result is due to de Branges and Rovnyak. It is of interest to find explicit examples where the hypothesis of Theorem [1.1 is in force, and hence the above trace formula is true. One such example is given by the case of canonical differential expressions; see $\sqrt[86]{6}$ and $[8]$. Another example is obtained when $G_{+}$and $G_{-}$ are extensions of a common Hermitian operator with deficiency indices $(n, n)$ (see 23 p. 420]). Then we use the notion of the $Q$-function of a closed Hermitian operator (due to Kreı̆; see [30, 31, 36, 42]). See \$7.

Proof of Theorem 1.1. We follow [20, pp. 330-333] and proceed in a number of steps.

Step 1. Let $\alpha \in \mathbb{C} \backslash \mathbb{R}$. The operator

$$
U=\left(G_{+}-\bar{\alpha} I\right)^{-1}\left(G_{+}-\alpha I\right)\left(G_{-}-\bar{\alpha} I\right)\left(G_{-}-\alpha I\right)^{-1}
$$

is unitary. If $\delta=I-U$, then $\operatorname{ker} \delta=\operatorname{ker} \delta^{*}$.

This follows from the fact that for any selfadjoint operator $A$ and any point $\alpha$ off the real line, the operator

$$
(A-\bar{\alpha} I)(A-\alpha I)^{-1} \stackrel{\text { def }}{=} I+(\alpha-\bar{\alpha})(A-\alpha I)^{-1}
$$

is unitary, as is easily seen by using, e.g., the spectral theorem. 
For the proof of the second claim, let $u \in \operatorname{ker} \delta$. Then $W u=u$, and since $W$ is unitary, we have $u=W^{*} u$, that is, $u \in \operatorname{ker} \delta^{*}$, whence $\operatorname{ker} \delta \subset \operatorname{ker} \delta^{*}$. The converse inclusion is proved by reading the preceding argument backwards.

Step 2. Set $x(\alpha)=\left(G_{+}-\alpha I\right)^{-1}-\left(G_{-}-\alpha I\right)^{-1}$. We have

$$
\operatorname{rank} \delta=\operatorname{rank} x(\bar{\alpha})=n \quad \text { for any } \alpha \in \mathbb{C} \backslash \mathbb{R} \text {. }
$$

We write

$$
\begin{aligned}
& U-I=\left(I+(\bar{\alpha}-\alpha)\left(G_{+}-\bar{\alpha} I\right)^{-1}\right)\left(I+(\alpha-\bar{\alpha})\left(G_{-}-\alpha I\right)^{-1}\right)-I \\
& =(\bar{\alpha}-\alpha)\left\{\left(G_{+}-\bar{\alpha} I\right)^{-1}-\left(G_{-}-\alpha I\right)^{-1}\right. \\
& \left.+(\alpha-\bar{\alpha})\left(G_{+}-\bar{\alpha} I\right)^{-1}\left(G_{-}-\alpha I\right)^{-1}\right\} \\
& =(\bar{\alpha}-\alpha)\left\{x(\bar{\alpha})+\left(G_{-}-\bar{\alpha} I\right)^{-1}-\left(G_{-}-\alpha I\right)^{-1}\right. \\
& \left.+(\alpha-\bar{\alpha})\left(G_{+}-\bar{\alpha} I\right)^{-1}\left(G_{-}-\alpha I\right)^{-1}\right\} \text {. }
\end{aligned}
$$

Since $\left(G_{-}-\bar{\alpha} I\right)^{-1}-\left(G_{-}-\alpha I\right)^{-1}=(\bar{\alpha}-\alpha)\left(G_{-}-\bar{\alpha} I\right)^{-1}\left(G_{-}-\alpha I\right)^{-1}$, we have

$$
\begin{aligned}
U-I= & (\bar{\alpha}-\alpha)\left\{x(\bar{\alpha})+(\bar{\alpha}-\alpha)\left(G_{-}-\bar{\alpha} I\right)^{-1}\left(G_{-}-\alpha I\right)^{-1}\right. \\
& \left.+(\alpha-\bar{\alpha})\left(G_{+}-\bar{\alpha} I\right)^{-1}\left(G_{-}-\alpha I\right)^{-1}\right\} \\
& =(\bar{\alpha}-\alpha)\left\{x(\bar{\alpha})+x(\bar{\alpha})(\alpha-\bar{\alpha})\left(G_{-}-\alpha I\right)^{-1}\right\} \\
& =(\bar{\alpha}-\alpha) x(\bar{\alpha})\left(I+(\alpha-\bar{\alpha})\left(G_{-}-\alpha I\right)^{-1}\right),
\end{aligned}
$$

and the result follows, because the operator $I+(\alpha-\bar{\alpha})\left(G_{-}-\alpha I\right)^{-1}$ is unitary.

Step 3. There exists an invertible matrix $N_{0} \in \mathbb{C}^{n \times n}$ and a bounded operator $C: \mathcal{H} \rightarrow$ $\mathbb{C}^{n}$ such that $\delta=(\bar{\alpha}-\alpha) C^{*} N_{0} C$.

By Step $1, \mathcal{H}=\operatorname{ran} \delta \oplus \operatorname{ker} \delta$. Let $P$ denote the orthogonal projection from $\mathcal{H}$ onto $\operatorname{ran} \delta$, and let $X$ be a unitary operator from $\operatorname{ran} \delta$ onto $\mathbb{C}^{n}$. Then

$$
\delta=P\left(\left.\delta\right|_{\operatorname{ran} \delta}\right) P=P X^{*} X\left(\left.\delta\right|_{\operatorname{ran} \delta}\right) X^{*} X P=C^{*} N_{0} C
$$

with

$$
C=X P \quad \text { and } \quad N_{0}=X\left(\left.\delta\right|_{\operatorname{ran} \delta}\right) X^{*}
$$

Step 4. Let

$$
C_{+}(z)=C\left(G_{+}-\alpha I\right)\left(G_{+}-z I\right)^{-1},
$$

and, for $h \in \mathcal{H}$, let $\widehat{h}(z)=C_{+}(z) f$. Then, $\widehat{h}(z) \equiv 0$ if and only if $h=0$. Furthermore,

$$
C_{+}(z)\left(\left(G_{+}-w I\right)^{-1} h\right)=R_{w} \widehat{h} .
$$

Assume that $C_{+}(z) h \equiv 0$. Then $C^{*} N_{0} C_{+}(z) h \equiv 0$, and so

$$
\begin{aligned}
\left\{I-\left(G_{+}-\bar{\alpha} I\right)^{-1}\left(G_{+}-\alpha I\right)\right. & \left.\left(G_{-}-\bar{\alpha} I\right)\left(G_{-}-\alpha I\right)^{-1}\right\} \\
& \times\left(G_{+}-\alpha I\right)\left(G_{+}-z I\right)^{-1} h \equiv 0 .
\end{aligned}
$$

Multiplying both sides from the left by the unitary operator $\left(G_{+}-\bar{\alpha} I\right)\left(G_{+}-\alpha I\right)^{-1}$, we obtain

$$
\begin{aligned}
\left\{\left(G_{+}-\bar{\alpha} I\right)\left(G_{+}-\alpha I\right)^{-1}-\right. & \left.\left(G_{-}-\bar{\alpha} I\right)\left(G_{-}-\alpha I\right)^{-1}\right\} \\
& \times\left(G_{+}-\alpha I\right)\left(G_{+}-z I\right)^{-1} h \equiv 0 .
\end{aligned}
$$

Relation (5.7) implies that

$$
\begin{array}{r}
\left\{\left(G_{+}-\bar{\alpha} I\right)\left(G_{+}-\alpha I\right)^{-1}-\left(G_{-}-\bar{\alpha} I\right)\left(G_{-}-\alpha I\right)^{-1}\right\} \\
=(\alpha-\bar{\alpha})\left\{\left(G_{+}-\alpha I\right)^{-1}-\left(G_{-}-\alpha I\right)^{-1}\right\} .
\end{array}
$$


Thus,

$$
\left(\left(G_{+}-\alpha I\right)^{-1}-\left(G_{-}-\alpha I\right)^{-1}\right)\left(G_{+}-\alpha I\right)\left(G_{+}-z I\right)^{-1} h \equiv 0,
$$

so that

$$
\begin{aligned}
\left(G_{+}\right. & -z I)^{-1} h \equiv\left(G_{-}-\alpha I\right)^{-1}\left(G_{+}-\alpha I\right)\left(G_{+}-z I\right)^{-1} h \\
& \equiv\left(G_{-}-\alpha I\right)^{-1}\left\{I+(z-\alpha)\left(G_{+}-z I\right)^{1}\right\} h \\
& \equiv\left(G_{-}-\alpha I\right)^{-1} h+(z-\alpha)\left(G_{-}-\alpha I\right)^{-1}\left(G_{+}-z I\right)^{-1} h .
\end{aligned}
$$

Consequently,

$$
\begin{gathered}
\left(G_{-}-\alpha I\right)^{-1} h \equiv\left(I-(z-\alpha)\left(G_{-}-\alpha I\right)^{-1}\right)\left(G_{+}-z I\right)^{-1} h \\
\equiv\left(G_{-}-z I\right)\left(G_{-}-\alpha I\right)^{-1}\left(G_{+}-z I\right)^{-1}
\end{gathered}
$$

whence $\left(G_{-}-z I\right)^{-1}\left(G_{-}-\alpha I\right)^{-1} h \equiv\left(G_{-}-\alpha I\right)^{-1}\left(G_{+}-z I\right)^{-1} h$. It follows that

$$
\left(G_{-}-z I\right)^{-1} h \equiv\left(G_{+}-z I\right)^{-1} h,
$$

and (5.3) shows that $h=0$.

Now we prove (5.10):

$$
\begin{aligned}
C_{+}(z) & \left\{\left(G_{+}-w I\right)^{-1} h\right\}=C\left(G_{+}-w I\right)\left\{\left(G_{+}-z I\right)^{-1}\left(G_{+}-w I\right)^{-1}\right\} h \\
= & C\left(G_{+}-w I\right)\left\{\frac{\left(G_{+}-z I\right)^{-1}-\left(G_{+}-w I\right)^{-1}}{z-w}\right\} h \\
= & \frac{C_{+}(z) h-C_{+}(w) h}{z-w} \\
= & R_{w}\left(C_{+}(z) h\right) .
\end{aligned}
$$

Step 5. Let

$$
N(z)=N_{0}^{-1}-(\alpha-z) C_{+}(z) C_{+}(\bar{\alpha})^{*} .
$$

Then

$$
\frac{N(z)-N(w)^{*}}{(z-\bar{w})}=C_{+}(z) C_{+}(w)^{*}
$$

First, we observe that

$$
\begin{gathered}
C_{+}(z) C_{+}(w)^{*}=C\left(I+(z-\alpha)\left(G_{+}-z I\right)^{-1}\right)\left(I+(\bar{w}-\bar{\alpha})\left(G_{+}-\bar{w}\right)^{-1}\right) C^{*} \\
=C\left\{I+(z-\alpha)\left(G_{+}-z I\right)^{-1}+(\bar{w}-\bar{\alpha})\left(G_{+}-\bar{w} I\right)^{-1}\right. \\
\left.\quad+\frac{(z-\alpha)(\bar{w}-\bar{\alpha})}{z-\bar{w}}\left(\left(G_{+}-z I\right)^{-1}-\left(G_{+}-\bar{w} I\right)^{-1}\right)\right\} C^{*} \\
=C\left\{I+(z-\alpha)\left(1+\frac{\bar{w}-\bar{\alpha}}{z-\bar{w}}\right)\left(G_{+}-z I\right)^{-1}\right. \\
\left.+(\bar{w}-\bar{\alpha})\left(1-\frac{z-\alpha}{z-\bar{w}}\right)\left(G_{+}-\bar{w} I\right)^{-1}\right\} C^{*},
\end{gathered}
$$

which in turn is equal to

$$
C\left\{\frac{z+(z-\alpha)(z-\bar{\alpha})\left(G_{+}-z I\right)^{-1}-\bar{w}-(\bar{w}-\alpha)(\bar{w}-\bar{\alpha})\left(G_{+}-\bar{w} I\right)^{-1}}{z-\bar{w}}\right\} C^{*} .
$$

Therefore,

$$
C_{+}(z) C_{+}(w)^{*}=\frac{N_{1}(z)-N_{1}(w)^{*}}{z-\bar{w}},
$$


where we have set

$$
N_{1}(z)=C\left(z I+(z-\alpha)(z-\bar{\alpha})\left(G_{+}-z I\right)^{-1}\right) C^{*} .
$$

Next we compute $\frac{N(z)-N(\bar{w})}{z-\bar{w}}$, where $N(z)$ is given by (5.11). We have

$$
\begin{gathered}
N(z)-N(\bar{w})=-C\left\{(\alpha-z)\left(G_{+}-\alpha I\right)\left(G_{+}-z I\right)^{-1}\right. \\
\left.\quad-(\alpha-\bar{w})\left(G_{+}-\alpha I\right)\left(G_{+}-\bar{w} I\right)^{-1}\right\}\left(G_{+}-\bar{\alpha}\right)\left(G_{+}-\alpha I\right)^{-1} C^{*} \\
=-C\left\{(\alpha-z)\left(G_{+}-z I\right)^{-1}\left(G_{+}-\bar{\alpha}\right)-(\alpha-\bar{w})\left(G_{+}-w I\right)^{-1}\left(G_{+}-\bar{\alpha} I\right)\right\} C^{*} \\
=-C\left\{(\alpha-z)\left(I+(z-\bar{\alpha})\left(G_{+}-z I\right)^{-1}\right)-(\alpha-\bar{w})\left(I+(w-\bar{\alpha})\left(G_{+}-\bar{w}\right)^{-1}\right)\right\},
\end{gathered}
$$

so that

$$
\begin{aligned}
N(z)-N(\bar{w})= & C\left\{z+(z-\alpha)(z-\bar{\alpha})\left(G_{+}-z I\right)^{-1}\right. \\
& \left.\quad-\bar{w}-(\bar{w}-\alpha)(w-\bar{\alpha})\left(G_{+}-\bar{w}\right)^{-1}\right\} C^{*} \\
= & N_{1}(z)-N_{1}(w)^{*} .
\end{aligned}
$$

Thus, $N(z)-N_{1}(z)$ is a constant. Consequently, $N(w)^{*}=N(w)^{*}$, and we obtain (5.12).

Formula (5.12) expresses the fact that the set of functions of the form $z \mapsto C_{+}(z) h$ with the norm $\left\|C_{+}(z) h\right\|=\|h\|_{\mathcal{H}}$ is a reproducing-kernel Hilbert space with the reproducing kernel given by the right-hand side of (5.12). The next steps of the proof consist in showing that this space $\mathcal{L}(N)$ has the required properties.

Step 6. Let $C_{-}=N_{0} C$. We have

$$
I-\left(G_{-}-\bar{\alpha} I\right)^{-1}\left(G_{-}-\alpha I\right)\left(G_{+}-\bar{\alpha} I\right)\left(G_{+}-\alpha I\right)^{-1}=C_{-}^{*} N_{0}^{-1} C_{-} .
$$

Indeed, the left-hand side of $\left(\underline{5.13)}\right.$ is equal to $I-U^{*}$, where $U$ is as in Step 1. From the identity $I-U=C^{*} N_{0} C$, we obtain

$$
I-U^{*}=C^{*} N_{0}^{*} C=C^{*} N_{0}^{*} N_{0}^{-1} N_{0} C=C_{-}^{*} N_{0}^{-1} C_{-} .
$$

Step 7. Let $C_{-}(z)=\frac{1}{4} C_{-}\left(G_{-}-\alpha I\right)\left(G_{-}-z I\right)^{-1}$, and let $\widetilde{h}(z)=C_{-}(z) h$. Then $C_{-}(z) h \equiv 0$ if and only if $h=0$. Furthermore, we have

$$
C_{-}(z)\left(\left(G_{-}-w I\right)^{-1} h\right)=R_{w} \widetilde{h} .
$$

The proof is similar to that in Step 6 and is omitted.

Step 8. We have

$$
N(z) C_{-}(z)=C_{+}(z)
$$

We write

$$
\begin{aligned}
N(z) C_{-} & (z)-C_{+}(z)=\left(N_{0}^{-1}+(z-\alpha) C_{+}(z) C_{+}(\bar{\alpha})^{*}\right) C_{-}(z)-C_{+}(z) \\
= & C\left(G_{-}-\alpha I\right)\left(G_{-}-z I\right)^{-1} \\
& +(z-\alpha)\left\{C\left(G_{+}-\alpha I\right)\left(G_{+}-z I\right)^{-1}\left(G_{+}-\alpha I\right)^{-1}\right. \\
& \left.\quad \times\left(G_{+}-\bar{\alpha} I\right)\left(G_{+}-\bar{\alpha} I\right) C^{*} N_{0} C\left(G_{-}-\alpha I\right)\left(G_{-}-z I\right)^{-1}\right\} \\
& -C\left(G_{+}-\alpha I\right)\left(G_{+}-z I\right)^{-1} \\
= & C\left(G_{-}-\alpha I\right)\left(G_{-}-z I\right)^{-1} \\
& +(z-\alpha)\left\{C\left(G_{+}-z I\right)^{-1}\left(G_{+}-\bar{\alpha} I\right) C^{*} N_{0} C\left(G_{-}-\alpha I\right)\left(G_{-}-z I\right)^{-1}\right\} \\
& -C\left(G_{+}-\alpha I\right)\left(G_{+}-z I\right)^{-1} .
\end{aligned}
$$


Replacing $\left(G_{ \pm}-\alpha I\right)\left(G_{ \pm}-z I\right)^{-1}$ by $I+(z-\alpha)\left(G_{ \pm}-z I\right)^{-1}$, and replacing $C^{*} N_{0} C$ by its value, we see that for the proof of (5.14) it suffices to check the identity

$$
\begin{aligned}
\pi(\bar{\alpha}-\alpha) & \left\{\left(G_{-}-z I\right)^{-1}-\left(G_{+}-z I\right)^{-1}\right\} \\
= & \left(G_{+}-z I\right)^{-1}\left(G_{+}-\bar{\alpha} I\right)\left(G_{-}-\alpha I\right)\left(G_{-}-z I\right)^{-1} \\
& -\left(G_{+}-z I\right)^{-1}\left(G_{+}-\alpha I\right)\left(G_{-}-\bar{\alpha} I\right)\left(G_{-}-z I\right)^{-1} .
\end{aligned}
$$

Here the right-hand side is equal to

$$
\begin{aligned}
& \left\{\left(I+(z-\bar{\alpha})\left(G_{+}-z I\right)^{-1}\right)\left(I+(z-\alpha)\left(G_{-}-z I\right)^{-1}\right)\right. \\
& \left.-\left(I+(z-\alpha)\left(G_{+}-z I\right)^{-1}\right)\left(I+(z-\bar{\alpha})\left(G_{-}-z I\right)^{-1}\right)\right\} \\
& \quad=(\alpha-\bar{\alpha})\left\{\left(G_{+}-z I\right)^{-1}-\left(G_{-}-z I\right)^{-1}\right\},
\end{aligned}
$$

whence the result.

More generally, with the preceding result at hand, one can rewrite all the remaining formulas in Theorem 4.3 We leave this to the reader.

Using the model and arguing as in our earlier paper [8, we arrive at the following corollary, which will be used in the proof of the trace formula.

Corollary 5.2. With the notation and under the hypothesis of Theorem 1.1, we have

$$
U_{+}\left\{\left(G_{+}-w I\right)^{-1}-\left(G_{-}-w I\right)^{-1}\right\} U_{+}^{*} F=\left(R_{w} N\right) N(w)^{-1} F(w)
$$

for $w \in \mathbb{C} \backslash \mathbb{R}$.

We note that, in the finite-dimensional case, (5.15) was proved in $\$ 2$ see (2.35).

Proof of Corollary 5.2. We have the formulas $U_{+}\left\{\left(G_{+}-w I\right)^{-1}\right\} U_{+}^{*} F=R_{w} F$. The commutativity condition (5.6) leads to

$$
\begin{aligned}
U_{+}\left(G_{-}-w I\right)^{-1} U_{+}^{*} F=\mathcal{M}_{N} U_{-}\left(G_{-}-w I\right)^{-1} U_{-}^{*} \mathcal{M}_{N}^{-1} F \\
\quad=\mathcal{M}_{N}\left(R_{w}\left(\mathcal{M}_{N}^{-1} F\right)\right) \\
\quad=N(z)\left(\frac{N^{-1}(z) F(z)-N^{-1}(w) F(w)}{z-w}\right) \\
=\frac{F(z)-N(z) N(w)^{-1} F(w)}{z-w} \\
=\frac{F(z)-F(w)}{z-w}-\frac{N(z)-N(w)}{z-w} N(w)^{-1} F(w) \\
=U_{+}\left(G_{+}-w I\right)^{-1} U_{+}^{*}-\frac{N(z)-N(w)}{z-w} N(w)^{-1} F(w),
\end{aligned}
$$

and the result follows.

5.2. A trace formula. Now we prove Theorem 1.2 As in the preceding section, first we repeat the theorem (with $G_{ \pm}$in place of $H_{ \pm}$).

Theorem 1.2. Let $\left(G_{+}, G_{-}\right)$be a pair of selfadjoint operators defined on a separable Hilbert space and satisfying the hypothesis of Theorem 1.1. Let $N$ be the associated Herglotz-Nevanlinna function. Then for all $w$ in the open upper half-plane we have

$$
\operatorname{Tr}\left\{\left(G_{+}-w I\right)^{-1}-\left(G_{-}-w I\right)^{-1}\right\}=\operatorname{Tr} N(w)^{-1} N^{\prime}(w)
$$

and

$$
\widetilde{\operatorname{det}_{z_{0}}}\left(G_{-}-z I\right)\left(G_{+}-z I\right)^{-1}=\operatorname{det} N(z) N\left(z_{0}\right)^{-1}, \quad z \in \mathbb{C}_{+},
$$

where $z_{0} \in \mathbb{C} \backslash \mathbb{R}$ and $\widetilde{\operatorname{det}}_{z_{0}}$ is the generalized perturbation determinant associated with the pair $\left(G_{+}, G_{-}\right)$. 
We begin with the trace formula (5.16). Recall that we have put

$$
x(w)=\left(G_{+}-w I\right)^{-1}-\left(G_{-}-w I\right)^{-1} .
$$

We proceed in a number of steps.

Step 1. Let $f \in \mathcal{H}$, and let $F=U_{+} f \in \mathcal{L}(N)$ and $w \in \mathbb{C} \backslash \mathbb{R}$. Then

$$
\langle x(w) f, f\rangle_{\mathcal{L}(N)}=\operatorname{Tr}\left\{N(w)^{-1} F(w) F(\bar{w})^{*}\right\} .
$$

Using Corollary 5.2 we obtain

$$
\langle f, x(w) f\rangle_{\mathcal{H}}=4 \pi\left\langle F, K_{N}(z, \bar{w}) N(w)^{-1} F(w)\right\rangle_{\mathcal{L}(N)}=4 \pi F(w)^{*} N(w)^{-*} F(\bar{w}),
$$

whence

$$
\langle x(w) f, f\rangle_{\mathcal{L}(N)}=F(\bar{w}) N(w)^{-1} F(w)=\operatorname{Tr}\left\{N(w)^{-1} F(w) F(\bar{w})^{*}\right\} .
$$

Step 2. Let $F_{1}, F_{2}, \ldots$ be an orthonormal basis of $\mathcal{L}(N)$. Then, in the norm of $\mathcal{L}(N)$ and pointwise, we have

$$
K_{N}(z, w)=\sum_{p=0}^{\infty} F_{p}(z) F_{p}(w)^{*} .
$$

This is a general result on reproducing kernels; see, e.g., [11, 43].

Step 3. Let $w \in \mathbb{C} \backslash \mathbb{R}$. Then

$$
N^{\prime}(w)=\left(\sum_{p=0}^{\infty} F_{p}(w) F_{p}(\bar{w})^{*}\right) .
$$

Plugging $\bar{w}$ in place of $w$ in (5.20) and taking the relation $N(\bar{w})=N(w)^{*}$ into account, we obtain

$$
\frac{N(z)-N(w)}{z-w}=K_{N}(z, \bar{w})=\left(\sum_{p=0}^{\infty} F_{p}(z) F_{p}(\bar{w})^{*}\right) .
$$

Letting $z \rightarrow w$ yields

$$
N^{\prime}(w)=\left(\sum_{p=0}^{\infty} F_{p}(w) F_{p}(\bar{w})^{*}\right)
$$

Step 4. We have

$$
\operatorname{Tr} x(w)=\operatorname{Tr} N(w)^{-1} N^{\prime}(w)=(\ln \operatorname{det} N)^{\prime}(w) .
$$

By the definition of the trace,

$$
\operatorname{Tr} x(w)=\sum_{p=0}^{\infty}\left\langle x(w) F_{p}, F_{p}\right\rangle_{\mathcal{L}(N)},
$$

where $\left\{F_{1}, F_{2}, \ldots\right\}$ is any orthonormal basis in $\mathcal{L}(N)$. Consequently,

$$
\operatorname{Tr} x(w)=\sum_{p=0}^{\infty} \operatorname{Tr}\left\{N(w)^{-1} F_{p}(w) F_{p}(\bar{w})^{*}\right\}=\operatorname{Tr} N(w)^{-1} N^{\prime}(w),
$$

where we have used the preceding step to get the second identity.

Finally, (5.17) is deduced from (5.16) as this was done in [8]. 
5.3. Inverse problems. Now we consider a general inverse problem associated with Herglotz-Nevanlinna functions. Two important special cases (for functions in the Wiener algebra and for $Q$-functions of Hermitian operators) will be considered in Subsections 6.3 and [7.3. respectively.

Theorem 5.3. A Herglotz-Nevanlinna function $N$ is the characteristic function of a pair of selfadjoint operators if and only if conditions (4.13) and (4.14) are fulfilled. Under these conditions, a pair $\left(G_{+}, G_{-}\right)$of operators for which $N$ is the characteristic function is obtained as follows:

$$
\begin{array}{ll}
G_{+} F(z)=z F(z)+c_{F}, & F \in \mathcal{L}(N), \\
G_{-} F(z)=z F(z)+c_{F}, & F \in \mathcal{L}\left(-N^{-1}\right) .
\end{array}
$$

The proof is similar to that of Theorem 4.9. Conditions (4.13) and (4.14) ensure that the spaces $\mathcal{L}(N)$ and $\mathcal{L}\left(-N^{-1}\right)$ contain no nonzero constant functions.

One can also express $G_{+}$and $G_{-}$in a Lebesgue space as in (4.16).

\section{$\S 6$. The Case of Canonical Differential expressions}

In this section we consider the case of the canonical differential expressions, which was treated in detail in our paper [8].

6.1. Preliminaries. Canonical differential expressions are differential equations of the form

$$
-i J \frac{\mathrm{d} f}{\mathrm{~d} t}(t, z)=z f(t, z)+V(t) f(t, z), \quad t \geq 0, \quad z \in \mathbb{R},
$$

where

$$
J=\left(\begin{array}{cc}
I_{n} & 0 \\
0 & -I_{n}
\end{array}\right), \quad V(t)=\left(\begin{array}{cc}
0 & k(t) \\
k(t)^{*} & 0
\end{array}\right),
$$

and the function $k$ (called the potential) is $\mathbb{C}^{n \times n}$-valued and with entries in $\mathbf{L}_{1}(0, \infty)$. The solution $f(t, z)$ is $\mathbb{C}^{2 n \times p}$-valued (typically, $p$ will be equal to $1, n$, or $2 n$ ). These expressions were introduced by M. G. Kreĭn (see, e.g., 34]), and we studied them in [4, 5] in the case where the scattering function is rational. It is of interest to relate precisely the properties of $k, S$, and the weight function $W$. This was done in [4], where we obtained an explicit expression for the potential in terms of a minimal realization of the spectral function or in terms of the scattering function. We studied such differential expressions in $9,10,4,5,6,6$, 7 .

The differential equation (6.1) has a unique $\mathbb{C}^{2 n \times 2 n}$-valued solution $\Theta(t, z)$ subject to the initial condition $\Theta(0, z)=I_{2 n}$.

Definition 6.1. The Weyl coefficient function $N(z)$ is defined in the open upper halfplane; it is a unique $\mathbb{C}^{n \times n}$-valued function such that

$$
\int_{0}^{\infty}\left(i N(z)^{*} I_{n}\right)\left(\begin{array}{cc}
I_{n} & I_{n} \\
I_{n} & -I_{n}
\end{array}\right) \Theta(t, z)^{*} \Theta(t, z)\left(\begin{array}{cc}
I_{n} & I_{n} \\
I_{n} & -I_{n}
\end{array}\right)\left(\begin{array}{c}
-i N(z) \\
I_{n}
\end{array}\right) d t<\infty
$$

for $\operatorname{Im} z>0$.

With the differential expression (6.1), we associate a pair of selfadjoint operators as follows: $G_{+}$is defined by

$$
G_{+} f(t)=-i J \frac{\mathrm{d} f}{\mathrm{~d} t}(t, z)-V(t) f(t, z)
$$


with the domain formed by the elements $f \in \mathbf{L}_{2}^{2 n}(0, \infty)$ such that $f^{\prime} \in \mathbf{L}_{2}^{2 n}(0, \infty)$, and $\left(I_{n}-I_{n}\right) f(0)=0$, and $G_{-}$is defined by

$$
G_{-} f(t)=-i J \frac{\mathrm{d} f}{\mathrm{~d} t}(t, z)+V(t) f(t, z)
$$

with the same domain.

Theorem 6.2. The pair $\left(G_{+}, G_{-}\right)$of selfadjoint operators defined by (6.2) and (6.3) satisfies the hypothesis of the de Branges-Rovnyak model. The associated HerglotzNevanlinna function $N$ that describes the model is the Weyl function associated with the expression (6.1).

6.2. The trace formula. The trace formula for pairs of differential operators takes the following form.

Theorem 6.3. Let $G_{ \pm}$be the differential operators defined by (6.2) and (6.3) with potentials $\pm k(t) \in \mathbf{L}_{1}^{n \times n}\left(\mathbb{R}_{+}\right)$. Then for any $z$ off the real line, the rank of the operator

$$
\left(G_{+}-z I\right)^{-1}-\left(G_{-}-z I\right)^{-1}
$$

is equal to $n$. Let $N$ be the associated Herglotz-Nevanlinna function. Then the operator $\left(G_{+}-z I\right)\left(G_{-}-z I\right)^{-1}-I$ has rank $n$, and

$$
\operatorname{Tr}\left(\left(G_{+}-z I\right)^{-1}-\left(G_{-}-z I\right)^{-1}\right)=\operatorname{Tr} N(z)^{-1} N^{\prime}(z)
$$

and

$$
\widetilde{\operatorname{det}_{z_{0}}}\left(G_{-}-z I\right)\left(G_{+}-z I\right)^{-1}=\operatorname{det} N(z) N\left(z_{0}\right)^{-1}, \quad z \in \mathbb{C}_{+} .
$$

In the above formula, $\widetilde{\operatorname{det}}_{z_{0}}$ denotes the generalized perturbation determinant associated with the pair $\left(G_{+}, G_{-}\right)$. For the proof, see 8 . The proof is based on the theory of reproducing-kernel Hilbert spaces of entire functions.

6.3. Inverse problems. If a given Herglotz-Nevanlinna function is the characteristic function of a pair of selfadjoint operators, then, in general, it seems difficult to give a concrete model for the pair, besides the one described in Subsections 2.6. 4.4, and 5.3 In the setting of the Wiener algebra, a specific model is available in terms of canonical differential expressions.

Theorem 6.4. A Herglotz-Nevanlinna function $N$ is the characteristic function of a pair of differential expressions $\left(G_{+}, G_{-}\right)$defined by (6.2), (6.3) if and only if $N$ belongs to the Wiener algebra $\mathcal{W}_{+}^{n \times n}$, satisfies $\operatorname{Im} N(t)>0$ for real $t$, and becomes equal to the identity at infinity.

The conditions of the theorem imply that $-N^{-1}$ is also in the Wiener algebra $\mathcal{W}_{+}^{n \times n}$ and is equal to the identity at infinity. Consequently, conditions (4.13) and (4.14) are fulfilled, as it should be.

Two equivalent strategies can be used to prove this theorem: Kreln's approach and Marchenko's approach. Under Kreln's approach, one starts with the spectral function $W(t)=\operatorname{Im} N(t)$, while under Marchenko's approach one starts with the scattering function. Relationships between these approaches were studied in [26] and, in the rational case, in [5]. Following KreĬn's approach, we let $N$ be the Weyl coefficient and set

$$
W(t)=\operatorname{Im} N(t)=I_{n}-\int_{\mathbb{R}} e^{i s t} k(s) d s .
$$

For every $T>0$, the operator that takes $f \in \mathbf{L}_{2}^{n}[0, T]$ to the function

$$
f(t)-\int_{0}^{T} k(t-u) f(u) d u
$$


is invertible, and its inverse is of the form

$$
f(t)+\int_{0}^{T} \Gamma_{T}(t, u) f(u) d u .
$$

Then the potential is given by the formula

$$
k(t)=-2 i \Gamma_{2 t}(0,2 t) .
$$

We refer the reader to our papers mentioned before for a discussion of Marchenko's approach and for the study of the relationship between the two approaches.

\section{§7. Extensions of a common Hermitian operator}

In [30] and 31], M. G. Kreln introduced the notion of the $Q$-function of a symmetric simple closed operator $S$. This function makes it possible to give a formula that describes the set of all compressed resolvents of the selfadjoint extensions of $S$; see 36, formula (2.7) on p. 200]. In this section we establish the relationship between the $Q$-function and the characteristic function of a pair of selfadjoint operators. The $Q$-function is not uniquely determined. The main result of the section is the following. Let $(n, n)$ be the deficiency indices of $S$. If the pair $\left(G_{+}, G_{-}\right)$of selfadjoint extensions satisfies

$$
\operatorname{dim} \operatorname{ran}\left\{\left(G_{+}-z I\right)^{-1}-\left(G_{-}-z I\right)^{-1}\right\}=n
$$

(in general, inequality may occur), then the function $z \mapsto N(\bar{z})^{*}$ (where $N$ is the corresponding characteristic function) is a $Q$-function of the symmetric operator.

7.1. The $Q$-function and Kreŭn's formula for generalized resolvents. In this section we recall the definition and main properties of the $Q$-function. Let $S$ be a symmetric (we shall also use the term Hermitian) simple closed operator in a Hilbert space $\mathcal{H}$. Simple means that $S$ has no invariant subspace on which it is selfadjoint. We recall that for any nonreal number $z$ the range $\operatorname{ran}(z I-S)$ is a closed subspace of $\mathcal{H}$. The dimensions of the spaces

$$
\mathcal{H} \ominus \operatorname{ran}(z I-S)
$$

are the same for all $z$ in the upper half-plane and all $z$ in the lower half-plane, and are called the deficiency indices of the operator. We assume that the deficiency indices are equal and finite. Let $A$ denote a selfadjoint extension of $S$ in a Hilbert space $\widetilde{\mathcal{H}} \supset \mathcal{H}$, and let $P$ denote the orthogonal projection from $\widetilde{\mathcal{H}}$ onto $\mathcal{H}$. The function $R(z)=$ $\left.P(A-z I)^{-1}\right|_{\mathcal{H}}$ is called a generalized resolvent of $S$. The set of all generalized resolvents is described by Kreln's formula. To present this formula, we need to recall the definition of a $Q$-function; see [35, 36]. In order to define a $Q$-function, we fix a point $z_{0} \in \mathbb{C} \backslash \mathbb{R}$ and let $A_{0}$ be a selfadjoint extension of $S$ in $\mathcal{H}$ (such an extension is called canonical). For every $z \in \mathbb{C} \backslash \mathbb{R}$, the operator

$$
I+\left(z-\overline{z_{0}}\right)\left(A_{0}-z I\right)^{-1}
$$

is a bijection from $\operatorname{ker}\left(S^{*}-z_{0} I\right)$ onto $\operatorname{ker}\left(S^{*}-z I\right)$. Let $\Gamma_{z_{0}}$ denote a bijection from $\mathbb{C}^{n}$ onto $\operatorname{ker}\left(S^{*}-\overline{z_{0}} I\right)$, and let

$$
\Gamma_{z_{0}}(z)=\left(A_{0}-z_{0}\right)\left(A_{0}-z I\right)^{-1} \Gamma_{z_{0}} .
$$

Any $\mathbb{C}^{n \times n}$-valued function $Q$ analytic in $\mathbb{C} \backslash \mathbb{R}$ and such that

$$
\frac{Q(z)-Q(\omega)^{*}}{z-\bar{\omega}}=\Gamma_{z_{0}}(\omega)^{*} \Gamma_{z_{0}}(z)
$$

is called a $Q$-function of the Hermitian operator $S$; see [36, §2, p. 199].

The relationship with $\mathcal{L}(N)$-spaces is established with the help of the following proposition. 
Proposition 7.1. Let $Q$ be a $Q$-function. Then $Q(z)=Q(\bar{z})^{*}$, and the kernel

$$
\frac{Q(\bar{z})^{*}-Q(\bar{w})}{z-\bar{w}}
$$

is positive in $\mathbb{C} \backslash \mathbb{R}$.

Proof. Relation (7.2) implies that $Q(z)=Q(\bar{z})^{*}$, and the result follows from the definition of a $Q$-function.

Not every Nevanlinna-Herglotz function is a $Q$-function. Necessary and sufficient conditions can be found in [36]; see Subsection 7.3. Let $B \in \mathbb{C}^{n \times n}$ be any selfadjoint matrix; we set $y_{0}=\operatorname{Im} z_{0}$. The function

$$
Q_{0}(z)=B-i y_{0} \Gamma_{z_{0}}^{*} \Gamma_{z_{0}}+\left(z-\overline{z_{0}}\right) \Gamma_{z_{0}}^{*}\left(A_{0}-z_{0} I\right)\left(A_{0}-z I\right)^{-1} \Gamma_{z_{0}}^{*}
$$

is a $Q$-function of the closed symmetric operator $S$; see [36, (2.2), p. 199] and [39. The set of all $Q$-functions of a given symmetric operator was described in [36, Satz 2.1, p. 201]. To present the result, we recall a definition: $T$ is called a generalized selfadjoint matrix if there is a projection $\widehat{P}$ such that

$$
T=\widehat{T} \widehat{P}+\infty(I-\widehat{P}),
$$

where $\widehat{T}$ is a selfadjoint matrix. The symbol $\infty$ in the above formula is interpreted as follows:

$$
\left(T+Q_{0}(z)\right)^{-1}=\widehat{P}\left(\widehat{T}+\widehat{P} Q_{0}(z) \widehat{P}\right)^{-1} \widehat{P} .
$$

Theorem 7.2. As above, let $Q_{0}$ be a $Q$-function of a closed simple Hermitian operator $S$ with equal deficiency indices. Then the set of all $Q$-functions of $S$ is given by the formula

$$
Q(z)=Q_{0}(z)-\left(Q_{0}(z)-Q_{0}\left(z_{0}\right)^{*}\right)\left(T+Q_{0}(z)\right)^{-1}\left(Q_{0}(z)-Q_{0}\left(z_{0}\right)\right)+B,
$$

where $T$ is a generalized selfadjoint matrix and $B$ is a selfadjoint matrix.

KreĬn's formula gives a description of all generalized resolvents of a given symmetric operator with equal deficiency indices. When specialized to canonical extensions (that is, within the original Hilbert space $\mathcal{H}$ ), it takes the following form.

Theorem 7.3. Let $S$ be a closed symmetric operator with finite and equal deficiency indices $(n, n)$. Let $G_{0}$ denote a fixed canonical selfadjoint extension of $S$, and let $Q(z)$ be a $Q$-function of $S$. Then the formula

$$
(H-z I)^{-1}=\left(G_{0}-z I\right)^{-1}-\Gamma_{z_{0}}(z) \widehat{P}(\widehat{P} Q(z) \widehat{P}+\widehat{T})^{-1} \widehat{P} \Gamma_{z_{0}}\left(z^{*}\right)^{*}
$$

describes the set of all resolvents of the canonical selfadjoint extensions of $S$, where $\widehat{P}$ is an orthogonal projection from $\mathbb{C}^{n}$ into itself, and $\widehat{T} \in \mathbb{C}^{\widehat{n} \times \widehat{n}}$ (with $\left.\widehat{n}=\operatorname{dim} \operatorname{ran} \widehat{P}\right)$ is a selfadjoint matrix.

Formula (17.3) shows that for any two canonical extensions $G_{+}$and $G_{-}$of $S$ we have

$$
\operatorname{dim} \operatorname{ran}\left(\left(G_{+}-w I\right)^{-1}-\left(G_{-}-w I\right)^{-1}\right) \leq n .
$$

This dimension is exactly equal to $n$ if $\widehat{P}=I_{n}$.

The main result of this section is as follows.

Theorem 7.4. Let $S$ be a simple closed Hermitian operator with equal and finite deficiency indices $(n, n)$. Let $G_{+}$and $G_{-}$be two canonical selfadjoint extensions of $S$ such that

$$
\operatorname{dim} \operatorname{ran}\left(\left(G_{+}-w I\right)^{-1}-\left(G_{-}-w I\right)^{-1}\right)=n .
$$

Let $N$ be the characteristic function of the pair. Then the function $z \mapsto N(\bar{z})^{*}$ is a $Q$-function of $S$. 
An important ingredient of the proof of this theorem is the following lemma, which is of independent interest.

Lemma 7.5. Let $S$ be a Hermitian operator, and let $G_{+}$and $G_{-}$be two extensions of $S$. Let $z \in \mathbb{C} \backslash \mathbb{R}$. Then

$$
\operatorname{ran}\left\{\left(G_{+}-z I\right)^{-1}-\left(G_{-}-z I\right)^{-1}\right\} \subset \mathcal{H} \ominus \operatorname{ran}(S-\bar{z} I) .
$$

Proof. Let $u \in \operatorname{Dom} S$ and $h \in \mathcal{H}$. Then

$$
\begin{aligned}
\langle(S- & \left.\bar{z} I) u,\left\{\left(G_{+}-z I\right)^{-1}-\left(G_{-}-z I\right)^{-1}\right\} h\right\rangle_{\mathcal{H}} \\
& =\left\langle(S-\bar{z} I) u,\left(G_{+}-z I\right)^{-1} h\right\rangle_{\mathcal{H}}-\left\langle(S-\bar{z} I) u,\left(G_{-}-z I\right)^{-1} h\right\rangle_{\mathcal{H}} \\
& =\left\langle\left(G_{+}-\bar{z} I\right) u,\left(G_{+}-z I\right)^{-1} h\right\rangle_{\mathcal{H}}-\left\langle\left(G_{-}-\bar{z} I\right) u,\left(G_{-}-z I\right)^{-1} h\right\rangle_{\mathcal{H}},
\end{aligned}
$$

where we have used the inclusions $S \subset G_{+}$and $S \subset G_{-}$. Therefore, we obtain

$$
\left\langle(S-\bar{z} I) u,\left\{\left(G_{+}-z I\right)^{-1}-\left(G_{-}-z I\right)^{-1}\right\} h\right\rangle_{\mathcal{H}}=0,
$$

that is,

$$
\operatorname{ran}(S-\bar{z} I) \subset \operatorname{ran}\left\{\left(G_{+}-z I\right)^{-1}-\left(G_{-}-z I\right)^{-1}\right\}^{\perp},
$$

whence the result.

The above lemma implies that

$$
\text { dim ran }\left\{\left(G_{+}-z I\right)^{-1}-\left(G_{-}-z I\right)^{-1}\right\} \leq n .
$$

If $G_{+} \neq G_{-}$and $n>1$, strict inequality may occur; it suffices to take $\widehat{P} \neq I$ in (7.3). We note that in [23, p. 420] inequality (7.5) was claimed to be an identity.

Proof of Theorem 7.4. We fix a point $z_{0} \in \mathbb{C} \backslash \mathbb{R}$ and set $\alpha=\overline{z_{0}}$ in the arguments in the proof of Theorem[1.1. The inclusion (7.4) and the hypotheses of the theorem imply that

$$
\operatorname{ran}\left\{\left(G_{+}-z_{0} I\right)^{-1}-\left(G_{-}-z_{0} I\right)^{-1}\right\}=\mathcal{H} \ominus \operatorname{ran}\left(S-\overline{z_{0}} I\right) .
$$

Thus, in (7.1) we can take $\Gamma_{z_{0}}=\left.C^{*}\right|_{\operatorname{ran}\left(S-\overline{z_{0}} I\right)}$, where $C$ is as in Step 3 of the proof of Theorem 1.1 Therefore,

$$
C_{+}(z)=\Gamma_{z_{0}}(\bar{z})^{*}
$$

where $C_{+}(z)$ is defined by (5.9). Now, the result follows if we compare (7.2) and (5.12).

7.2. Trace formula: the case of common extensions of Hermitian operators. In the present setting the trace formula is easy to obtain. More precisely, taking the trace of Krĕn's formula, we arrive at the following result.

Theorem 7.6. Let $S$ be a simple closed symmetric operator with finite and equal deficiency indices $(n, n)$, and let $G_{+}$and $G_{-}$be two canonical extensions of $S$. Then there exists a $Q$-function of $S$ such that

$$
\operatorname{Tr}\left(\left(G_{+}-z I\right)^{-1}-\left(G_{-}-z I\right)^{-1}\right)=\operatorname{Tr} Q(z)^{-1} Q^{\prime}(z), \quad z \in \mathbb{C}_{+} .
$$

Proof. We take $G_{0}=G_{-}$in (7.3) and take a $Q$-function coming from $G_{-}$. The extension $G_{+}$corresponds to $\widehat{P}=I$ and a constant $T$. From (7.2) we see that

$$
\operatorname{Tr} \Gamma_{z_{0}}\left(z^{*}\right)^{*} \Gamma_{z_{0}}(z)=Q^{\prime}(z) \text {. }
$$

Hence, taking the trace of both sides in (7.3), we obtain

$$
\operatorname{Tr}\left(\left(G_{+}-z I\right)^{-1}-\left(G_{-}-z I\right)^{-1}\right)=\operatorname{Tr}(Q(z)+T)^{-1} Q^{\prime}(z), \quad z \in \mathbb{C}_{+},
$$

which leads to the required result by replacing $Q$ by $Q+T$ since $Q+T$ is still a $Q$-function of $S$. 
7.3. Inverse problems. A $Q$-function has a positive imaginary part in the upper halfplane, i.e., it is a Herglotz-Nevanlinna function. Krĕn and Langer proved a general converse result in the setting of operators in Pontryagin spaces. See [36, Hauptsatz, p. 203]. Specialized to the case of a Hilbert space and finite deficiency indices, their result reads as follows.

Theorem 7.7. $A \mathbb{C}^{n \times n}$-valued Nevanlinna function is a $Q$-function of a Hermitian operator with deficiency indices $(n, n)$ if and only if for every $e \in \mathbb{C}^{n}$ the following two conditions are fulfilled:

1) $\lim _{y \rightarrow \infty} \frac{e^{*} Q(i y) e}{y}=0$;

2) $\lim _{y \rightarrow \infty} y e^{*}(\operatorname{Im} Q(i y)) e=\infty$.

The first condition expresses the fact that the space $\mathcal{L}(Q)$ contains no nonzero constant functions; see Proposition [3.6. The second expresses the fact that the measure $d \mu$ in the Riesz-Herglotz representation of $Q$ is not summable. In particular, the operator of multiplication by the independent variable is unbounded in $\mathbf{L}_{2}^{n}(d \mu)$, so that the selfadjoint operator $G_{+}$defined by $\left(G_{+}-z I\right)^{-1}=R_{z}$ is unbounded in $\mathcal{L}(Q)$. Proposition 3.4 shows that $-Q(z)^{-1}$ is also a $Q$-function.

As was remarked in [36], the second condition also implies that $\operatorname{Im} Q(z)>0$ for at least one point in $\mathbb{C}_{+}$.

It is of interest to compare these conditions with conditions 4.13) and (4.14). The first is precisely (4.13). The second implies (4.14) but is not equivalent to it.

The Hermitian operator associated with a $Q$-function is given in the next theorem; see [36. Satz 5.1, p. 222].

Theorem 7.8. For a given $Q$-function $Q$, the associated operator $S$ is the closure of the operator $S_{0}$ defined as follows:

$$
\operatorname{Dom} S_{0}=\left\{F(z)=\sum_{w} K_{Q}(z, w) c_{w}: \sum_{w} Q(w)^{*} c_{w}=\sum_{w} c_{w}=0\right\},
$$

where all the sums are finite, and

$$
S_{0} F(z)=z F(z), \quad F \in \operatorname{Dom} S_{0} .
$$

In conclusion, we mention the following statement.

Theorem 7.9. Let $Q$ be $a \mathbb{C}^{n \times n}$-valued Herglotz-Nevanlinna function satisfying the conditions of Theorem 7.7. Then it is the characteristic function of the pair $\left(G_{+}, G_{-}\right)$of operators obtained as follows:

$$
\begin{array}{ll}
G_{+} F(z)=z F(z)+c_{F}, & F \in \mathcal{L}(Q), \\
G_{-} F(z)=z F(z)+c_{F}, & F \in \mathcal{L}\left(-Q^{-1}\right),
\end{array}
$$

where $c_{F}=-\lim _{z \rightarrow \infty} z F(z)$.

As before, here one can express $G_{+}$and $G_{-}$in the Lebesgue space $\mathbf{L}_{2}^{n}(d \mu)$, as in (4.16). We also note that in the special case where $N \in \mathcal{W}_{+}^{n \times n}$ the operators $G_{+}$and $G_{-}$can be chosen to be canonical differential expressions, as was explained in the preceding section and in [8].

\section{REFERENCES}

[1] D. Alpay, A. Dijksma, and H. Langer, Classical Nevanlinna-Pick interpolation with real interpolation points, Operator Theory and Interpolation (Bloomington, IN, 1996), Oper. Theory Adv. Appl., vol. 115, Birkhäuser, Basel, 2000, pp. 1-50. MR 1766806|(2001g:47026)

[2] D. Alpay and H. Dym, Hilbert spaces of analytic functions, inverse scattering, and operator models. I, Integral Equations Operator Theory 7 (1984), 589-641. MR0766625|(87h:47022a) 
[3] D. Alpay and I. Gohberg, Unitary rational matrix functions, Topics in Interpolation Theory of Rational Matrix-Valued Functions, Oper. Theory Adv. Appl., vol. 33, Birkhäuser, Basel, 1988, pp. 175-222. MR0960699 (90m:47006)

[4] _ Inverse spectral problem for differential operators with rational scattering matrix functions, J. Differential Equations 118 (1995), 1-19. MR1329400 (96f:34121)

[5] _ Inverse scattering problem for differential operators with rational scattering matrix functions, Singular Integral Operators and Related Topics (Tel Aviv, 1995), Oper. Theory Adv. Appl., vol. 90, Birkhäuser, Basel, 1996, pp. 1-18. MR1413548 (97j:34112)

[6] _ A relationship between the Nehari and the Carathéodory-Toeplitz extension problem, Integral Equations Operator Theory 26 (1996), 249-272. MR.1415031 (98c:47017)

[7] _ Inverse problems associated to a canonical differential system, Recent Advances in Operator Theory and Related Topics (Szeged, 1999), Oper. Theory Adv. Appl., vol. 127, Birkhäuser, Basel, 2001, pp. 1-27. MR1902792 (2003f:34158)

[8] — A trace formula for canonical differential expressions, J. Funct. Anal. 197 (2003), 489-525. MR 1960423 (2004a:47052)

[9] D. Alpay, I. Gohberg, M. A. Kaashoek, and A. L. Sakhnovich, Direct and inverse scattering problem for canonical systems with a strictly pseudo-exponential potential, Math. Nachr. 215 (2000), 5-31. MR,1768201 (2001i:34139)

[10] D. Alpay, I. Gohberg, and L. Sakhnovich, Inverse scattering problem for continuous transmission lines with rational reflection coefficient function, Recent Developments in Operator Theory and its Applications (Winnipeg, MB, 1994), Oper. Theory Adv. Appl., vol. 87, Birkhäuser, Basel, 1996, pp. 1-16. MR.1399354 (97f:34072)

[11] N. Aronszajn, Theory of reproducing kernels, Trans. Amer. Math. Soc. 68 (1950), 337-404. MR.0051437(14:479c)

[12] N. Aronszajn and W. F. Donoghue, A supplement to the paper on exponential representations of analytic functions in the upper half-plane with positive imaginary part, J. Anal. Math. 12 (1964), 113-127. MR0168769 (29:6025)

[13] _ On exponential representations of analytic functions in the upper half-plane with positive imaginary part, J. Anal. Math. 5 (1956/1957), 321-388.

[14] H. Bart, I. Gohberg, and M. Kaashoek, Minimal factorization of matrix and operator functions, Oper. Theory Adv. Appl., vol. 1, Birkhäuser, Basel-Boston, MA, 1979. MR0560504 (81a:47001)

[15] S. V. Belyi and E. R. Tsekanovskii, Realization theorems for operator-valued R-functions, New Results in Operator Theory and its Applications, Oper. Theory Adv. Appl., vol. 98, Birkhäuser, Basel, 1997, pp. 55-91. MR1478466 (98k:47018)

[16] - On classes of realizable operator-valued R-functions, Operator Theory and Interpolation (Bloomington, IN, 1996), Oper. Theory Adv. Appl., vol. 115, Birkhäuser, Basel, 2000, pp. 85-112. MR.1766808 (2001g:47028)

[17] M. Sh. Birman and D. R. Yafaev, The spectral shift function. The work of M. G. Kreun and its further development, Algebra i Analiz 4 (1992), no. 5, 1-44; English transl., St. Petersburg Math. J. 4 (1993), no. 5, 833-870. MF,1202723 (94g:47002)

[18] L. de Branges, Perturbations of selfadjoint transformations, Amer. J. Math. 84 (1962), 543-560. MR.0154132 (27:4083)

[19] _ Espaces Hilbertiens de fonctions entières, Masson, Paris, 1972. MR0390739 (52:11562)

[20] L. de Branges and J. Rovnyak, Canonical models in quantum scattering theory, Perturbation Theory and its Applications in Quantum Mechanics (Madison, Wis., 1965), Wiley, New York, 1966, pp. 295392. MR0244795 (39:6109)

[21] _ Square summable power series, Holt, Rinehart and Winston, New York, etc., 1966. MR.0215065 (35:5909)

[22] R. W. Carey, A unitary invariant for pairs of selfadjoint operators, J. Reine Angew. Math. 283/284 (1976), 294-312. MR0415366 (54:3454)

[23] J. Dieudonné, Éléments d'analyse. Tome 2: Chapitres XII à XV, Gauthier-Villars, Paris, 1968. MR.0235946 (38:4247)

[24] W. F. Donoghue, Monotone matrix functions and analytic continuation, Grundlehren Math. Wiss., vol. 207, Springer-Verlag, New York-Heidelberg, 1974. MR.0486556 (58:6279)

[25] H. Dym, J-contractive matrix functions, reproducing-kernel Hilbert spaces and interpolation, CBMS Regional Conf. Ser. Math., vol. 71. Published for the Conference Board of the Mathematical Sciences, Amer. Math. Soc., Providence, RI, 1989. MR1004239 (90g:47003)

[26] H. Dym and A. Iacob, Positive definite extensions, canonical equations, and inverse problems, Topics in Operator Theory Systems and Networks (Rehovot, 1983), Oper. Theory Adv. Appl., vol. 12, Birkhäuser, Basel, 1984, pp. 141-240. MRMR0761362 (86g:34025) 
[27] F. Gesztesy, K. A. Makarov, and S. N. Naboko, The spectral shift operator, Mathematical Results in Quantum Mechanics (Prague, 1998), Oper. Theory Adv. Appl., vol. 108, Birkhäuser, Basel, 1999, pp. 59-90. MR 1708788 (2000k:47012)

[28] I. Gohberg, P. Lancaster, and L. Rodman, Matrices and indefinite scalar products, Oper. Theory Adv. Appl., vol. 8, Birkhäuser, Basel, 1983. MR0859708 (87j:15001)

[29] M. Karow, Selfadjoint operators and pairs of Hermitian forms over the quaternions, Linear Algebra Appl. 299 (1999), no. 1-3, 101-117. MR 1723711 (2001c:15021)

[30] M. G. Krel̆n, On Hermitian operators with deficiency indices equal to one, Dokl. Akad. Nauk SSSR 43 (1944), 339-342. (Russian) MF0011170 (6:131a)

[31] , On resolvents of a Hermitian operator with deficiency indices $(m, m)$, Dokl. Akad. Nauk SSSR 52 (1946), no. 8, 657-660. (Russian) MR0018341 (8:277a)

[32] _ Topics in differential and integral equations and operator theory, Oper. Theory Adv. Appl., vol. 7, Birkhäuser Verlag, Basel-Boston, MA, 1983. MR0815109 (86m:00014)

[33] _ On the trace formula in perturbation theory, Mat. Sb. 33 (1953), no. 3, 597-626. (Russian) MR0060742 (15:720b)

[34] , Continual analogs of propositions for polynomials orthogonal on the unit circle, Dokl. Akad. Nauk SSSR 105 (1955), no. 4, 637-640. (Russian) MR0080735 (18:291b)

[35] M. G. Krel̆n and H. Langer, Über die verallgemeinerten Resolventen und die charakteristische Funktion eines isometrischen Operators im Raume $\pi_{k}$, Hilbert Space Operators and Operator Algebras (Proc. Internat. Conf., Tihany, 1970), Colloq. Math. Soc. Janos Bolyai, vol. 5, NorthHolland, Amsterdam, 1972, pp. 353-399. MR0423122 |(54:11103)

[36] _ Über die Q-Funktion eines $\pi$-hermiteschen Operators im Raume $\pi_{\kappa}$, Acta Sci. Math. (Szeged) 34 (1973), 191-230. MR0318958 (47:7504)

[37] M. G. Kreĭn and A. A. Nudel'man, The Markov moment problem and extremal problems. Ideas and problems of P. L. Chebyshev and A. A. Markov and their further development, "Nauka", Moscow, 1973; English transl., Transl. Math. Monogr., vol. 50, Amer. Math. Soc., Providence, RI, 1977. MR.0445244 (56:3588) MR0458081 (56:16284)

[38] M. G. Krĕn and V. A. Yavryan, Spectral shift functions that arise in perturbations of a positive operator, J. Operator Theory 6 (1981), no. 1, 155-191. MR.0637009 (83c:47023)

[39] H. Langer and B. Textorius, On generalized resolvents and $Q$-functions of symmetric linear relations (subspaces) in Hilbert space, Pacific J. Math. 72 (1977), no. 1, 135-165. MR0463964 (57:3902)

[40] I. M. Lifshits, On a problem of perturbation theory related to quantum statistics, Uspekhi Mat. Nauk 7 (1952), no. 1, 171-180. (Russian) MR0049490(14:185b)

[41] _ Some problems of the dynamic theory of non-ideal crystal lattices, Nuovo Cimento (10) 3 (1956), supplemento, 716-734. MR0085793 (19:85a)

[42] Sh. N. Saakyan, Theory of resolvents of a symmetric operator with infinite defect numbers, Dokl. Akad. Nauk Armyan. SSR 41 (1965), no. 4, 193-198. (Russian) MR.0196497 (33:4684)

[43] S. Saitoh, Theory of reproducing kernels and its applications, Pitman Res. Notes in Math., vol. 189, Longman Sci. Techn., Harlow; John Wiley and Sons, Inc., New York, 1988. MR0983117 (90f:46045)

[44] T. A. Springer and R. Steinberg, Conjugacy classes, Seminar on Algebraic Groups and Related Finite Groups (The Institute for Advanced Study, Princeton, NJ, 1968/69), Lecture Notes in Math., vol. 131, Springer, Berlin, 1970, pp. 167-266. MR0268192(42:3091)

Department of Mathematics, Ben-Gurion University of the Negev, POB 653, Beer-Sheva 84105 , ISRAEL

E-mail address: dany@math.bgu.ac.il

School of Mathematical Sciences, The Raymond and Beverly Sackler Faculty of Exact Sciences, Tel-Aviv University, Tel-Aviv, Ramat-Aviv 69989, Israel

E-mail address: gohberg@post.tau.ac.il

Received 24/OCT/2003 